\title{
AN EQUICONSISTENCY RESULT ON PARTIAL SQUARES
}

\author{
JOHN KRUEGER AND ERNEST SCHIMMERLING
}

\begin{abstract}
We prove that the following two statements are equiconsistent: there exists a greatly Mahlo cardinal; there exists a regular uncountable cardinal $\kappa$ such that no stationary subset of $\kappa^{+} \cap \operatorname{cof}(\kappa)$ carries a partial square.
\end{abstract}

A famous theorem in set theory is the result that the failure of the square principle $\square_{\kappa}$, for a regular uncountable cardinal $\kappa$, is equiconsistent with a Mahlo cardinal. Solovay proved that if $\lambda>\kappa$ is a Mahlo cardinal, then in any generic extension by the Lévy collapse $\operatorname{CoLL}(\kappa,<\lambda), \lambda=\kappa^{+}$and $\neg \square_{\kappa}$. On the other hand, Jensen [6] proved that $\neg \square_{\kappa}$ implies that $\kappa^{+}$is Mahlo in $L$.

Partial square sequences were introduced by Shelah as a weakening of the square principle. Let $\nu<\kappa^{+}$be regular, and let $A \subseteq \kappa^{+} \cap \operatorname{cof}(\nu)$. We say that $A$ carries a partial square if there exists a sequence $\left\langle c_{\alpha}: \alpha \in A\right\rangle$ satisfying: (a) $c_{\alpha}$ is a club subset of $\alpha$; (b) ot $\left(c_{\alpha}\right)=\nu$; (c) if $\gamma$ is a limit point of $c_{\alpha}$ and $c_{\beta}$, then $c_{\alpha} \cap \gamma=c_{\beta} \cap \gamma$.

A significant difference between the square principle and partial squares is that, while $\square_{\kappa}$ is independent of ZFC, the existence of partial squares is provable in ZFC. For example, Shelah [12] proved that if $\kappa$ is a regular uncountable cardinal, then $\kappa^{+} \cap \operatorname{cof}(<\kappa)$ splits into $\kappa$ many pairwise disjoint subsets each of which carries a partial square.

Another difference is that, unlike the square principle $\square_{\kappa}$, partial squares on $\kappa^{+} \cap \operatorname{cof}(\kappa)$ are consistent with $\kappa$ being supercompact. For example, suppose $\kappa$ is indestructibly supercompact. Then the forcing poset for adding a partial square sequence on the set $\kappa^{+} \cap \operatorname{cof}(\kappa)$ with initial segments is $\kappa$-directed closed and thus preserves the supercompactness of $\kappa$. Also if $V=L[E]$ is an extender model, then for any regular uncountable cardinal $\kappa, \kappa^{+} \cap \operatorname{cof}(\kappa)$ carries a partial square. On the other hand, if $\kappa$ is $\kappa^{+}$-supercompact (or even subcompact), then $\square_{\kappa}$ fails ([14], [3]).

Magidor [8] constructed a model of set theory which satisfies a strong form of stationary set reflection, using a weakly compact cardinal. In this model there is no stationary subset of $\omega_{2} \cap \operatorname{cof}\left(\omega_{1}\right)$ which carries a partial square. In [7] we define a forcing iteration which destroys the stationarity of any subset of $\kappa^{+} \cap \operatorname{cof}(\kappa)$ which carries a partial square, using a weakly compact cardinal. In this paper we show that the same forcing iteration works assuming only a greatly Mahlo cardinal.

We also obtain the lower bound, by showing that if no stationary subset of $\kappa^{+} \cap \operatorname{cof}(\kappa)$ carries a partial square, then $\kappa^{+}$is greatly Mahlo in $L$. Thus we prove the following equiconsistency result.

Theorem 1. The statement that there exists a regular uncountable cardinal $\kappa$ such that no stationary subset of $\kappa^{+} \cap \operatorname{cof}(\kappa)$ carries a partial square is equiconsistent with a greatly Mahlo cardinal.

Date: December 2010.

2010 Mathematical Subject Classification: 03E35, 03E45.

Key words and phrases: partial square sequence, greatly Mahlo cardinal. 
We now outline the contents of the paper. In Section 1 we review the basic facts about canonical functions and the Mahlo hierarchy which will be used in the paper. In Section 2 we prove that for a regular uncountable cardinal $\kappa$, if $\kappa^{+}$is not greatly Mahlo in $L$, then there exists a stationary subset of $\kappa^{+} \cap \operatorname{cof}(\kappa)$ which carries a partial square. The remainder of the paper shows how to force a model in which there are no partial squares using a greatly Mahlo cardinal. In Section 3 we review some facts about elementary substructures and the Lévy collapse. In Section 4 we define the idea of a partial square killing forcing iteration, and prove an absoluteness result. Section 5 describes some of the basic properties of this kind of iteration. Section 6 shows that after Lévy collapsing a greatly Mahlo cardinal, a partial square killing forcing iteration is distributive. In Section 7 we put the pieces together to prove the consistency result. Section 8 describes a related equiconsistency result.

Our notation is standard unless noted otherwise. We assume that the reader has some familiarity with $L$, but not necessarily with fine structure. We also assume that the reader understands the basics of forcing, iterated forcing, proper forcing, and the Lévy collapse, and is familiar with stationary subsets of $P_{\lambda}(X)=\{a \subseteq X$ : $|a|<\lambda\}$, where $\lambda$ is regular and uncountable ([1], [5], [13]).

\section{Canonical Functions and the Mahlo Hierarchy}

We work out the details about canonical functions which will be used in the paper, and define the Mahlo hierarchy. Most of this material is folklore; also see [2] and [4].

Let $\lambda$ be a regular uncountable cardinal. For functions $f, g: \lambda \rightarrow \lambda$, we write $f={ }^{*} g$ if there is a club $C \subseteq \lambda$ such that $f(\alpha)=g(\alpha)$ for all $\alpha$ in $C$. Clearly $={ }^{*}$ is an equivalence relation.

Definition 1.1. Let $\lambda$ be a regular uncountable cardinal, and let $\nu<\lambda^{+}$. $A$ function $f: \lambda \rightarrow \lambda$ is said to be a canonical function on $\lambda$ of rank $\nu$ if there exists a surjective function $g: \lambda \rightarrow \nu$ and a club $C \subseteq \lambda$ such that

$$
\forall \alpha \in C(f(\alpha)=\operatorname{ot}(g[\alpha])) \text {. }
$$

If $f_{1}$ is a canonical function on $\lambda$ of rank $\nu$ and $f_{1}={ }^{*} f_{2}$, then $f_{2}$ is a canonical function on $\lambda$ of rank $\nu$. Also note that if $g, h: \lambda \rightarrow \nu$ are surjective functions, then there are club many $\alpha$ such that $g[\alpha]=h[\alpha]$. It follows that if $f_{1}$ and $f_{2}$ are both canonical functions of rank $\nu$, then $f_{1}={ }^{*} f_{2}$. Hence the set of all canonical functions of rank $\nu$ is an equivalence class modulo $=^{*}$.

Notation 1.2. Let $\lambda$ be a regular uncountable cardinal and let $\nu<\lambda^{+}$. Then $\mathbf{f}_{\nu}^{\lambda}$ denotes the class of all canonical functions on $\lambda$ of rank $\nu$.

Clearly $\mathbf{f}_{\nu}^{\lambda}$ is non-empty for all $\nu<\lambda^{+}$. Note that $f \in \mathbf{f}_{0}^{\lambda}$ iff $f(\alpha)=0$ for club many $\alpha$.

Lemma 1.3. Let $\lambda$ be a regular uncountable cardinal, let $\nu_{0}<\nu_{1}<\lambda^{+}$, and let $g: \lambda \rightarrow \nu_{1}$ be a surjection. Fix $f_{\nu_{0}} \in \mathbf{f}_{\nu_{0}}^{\lambda}$. Then there is a club $C \subseteq \lambda$ such that

$$
\forall \alpha \in C\left(f_{\nu_{0}}(\alpha)=\operatorname{ot}\left(g[\alpha] \cap \nu_{0}\right)\right)
$$


Proof. If $\nu_{0}=0$, then the statement is clear. So assume $\nu_{0}>0$. Define a surjective function $h: \lambda \rightarrow \nu_{0}$ by

$$
h(\alpha)= \begin{cases}g(\alpha) & \text { if } g(\alpha) \in \nu_{0} \\ 0 & \text { otherwise }\end{cases}
$$

Fix $\gamma<\lambda$ such that $g(\gamma)=0$. Fix a club $C$ such that

$$
\forall \alpha \in C\left(\alpha>\gamma \wedge f_{\nu_{0}}(\alpha)=\operatorname{ot}(h[\alpha])\right) .
$$

Then for all $\alpha$ in $C, h[\alpha]=g[\alpha] \cap \nu_{0}$, and hence $f_{\nu_{0}}(\alpha)=\operatorname{ot}(h[\alpha])=\operatorname{ot}\left(g[\alpha] \cap \nu_{0}\right)$.

In particular, suppose $\nu_{0}<\nu_{1}<\lambda^{+}$and $g: \lambda \rightarrow \nu_{1}$ is a surjection. If we define $f: \lambda \rightarrow \lambda$ by letting $f(\alpha)=\operatorname{ot}\left(g[\alpha] \cap \nu_{0}\right)$ for all $\alpha$, then $f \in \mathbf{f}_{\nu_{0}}^{\lambda}$.

It is not hard to show using the last lemma that if $f_{\gamma}$ and $f_{\gamma+1}$ are in $\mathbf{f}_{\gamma}^{\lambda}$ and $\mathbf{f}_{\gamma+1}^{\lambda}$ respectively, then for club many $\alpha, f_{\gamma+1}(\alpha)=f_{\gamma}(\alpha)+1$.

If $\delta<\lambda^{+}$is a limit ordinal and $g: \lambda \rightarrow \delta$ is a surjection, then there is a club of $\alpha$ such that for all $\beta<\alpha$, there is $\gamma<\alpha$ such that $g(\beta)<g(\gamma)$. It follows that if $f_{\delta} \in \mathbf{f}_{\delta}^{\lambda}$, then $f_{\delta}(\alpha)$ is a limit ordinal for club many $\alpha$.

Given functions $f, g: \lambda \rightarrow \lambda$, we write $f<^{*} g$ to mean that there exists a club $C \subseteq \lambda$ such that $f(\alpha)<g(\alpha)$ for all $\alpha$ in $C$. Define $f \leq^{*} g$ similarly. If $f_{1}={ }^{*} f_{2}$ and $g_{1}=^{*} g_{2}$, then $f_{1}<^{*} g_{1}\left(f_{1} \leq^{*} g_{1}\right)$ implies $f_{2}<^{*} g_{2}\left(f_{2} \leq^{*} g_{2}\right)$.

Proposition 1.4. Let $\lambda$ be a regular uncountable cardinal, and let $\nu<\lambda^{+}$. Let $f_{\nu}$ be in $\mathbf{f}_{\nu}^{\lambda}$. Then $f_{\beta}<^{*} f_{\nu}$ for all $\beta<\nu$ and $f_{\beta} \in \mathbf{f}_{\beta}^{\lambda}$.

Proof. Fix a surjective function $g: \lambda \rightarrow \nu$ and a club $E$ such that for all $\alpha$ in $E$, $f_{\nu}(\alpha)=\operatorname{ot}(g[\alpha])$. Let $\beta<\nu$ and $f_{\beta} \in \mathbf{f}_{\beta}^{\lambda}$. Fix $\zeta<\lambda$ such that $g(\zeta)=\beta$. By Lemma 1.3, fix a club $C \subseteq \lambda$ such that for all $\alpha$ in $C, \alpha>\zeta$ and $f_{\beta}(\alpha)=\operatorname{ot}(g[\alpha] \cap \beta)$. Then for all $\alpha$ in $C \cap E, f_{\beta}(\alpha)=\operatorname{ot}(g[\alpha] \cap \beta)<\operatorname{ot}(g[\alpha] \cap \beta)+1=\operatorname{ot}((g[\alpha] \cap \beta) \cup\{\beta\}) \leq$ $\operatorname{ot}(g[\alpha])=f_{\nu}(\alpha)$.

The next proposition is another basic result about canonical functions; we omit the proof since we will not use this fact.

Proposition 1.5. Let $\lambda$ be a regular uncountable cardinal, and let $\nu<\lambda^{+}$. Let $f_{\beta}$ be in $\mathbf{f}_{\beta}^{\lambda}$ for all $\beta \leq \nu$. If $f: \lambda \rightarrow \lambda$ and $f_{\beta}<^{*} f$ for all $\beta<\nu$, then $f_{\nu} \leq^{*} f$.

It is straightforward to prove by induction using the proposition that for all $\nu<\lambda$, if $f_{\nu} \in \mathbf{f}_{\nu}^{\lambda}$, then $f_{\nu}(\alpha)=\nu$ for club many $\alpha$. Also if $f_{\lambda} \in \mathbf{f}_{\lambda}^{\lambda}$, then $f(\alpha)=\alpha$ for club many $\alpha$.

Next we relate the canonical functions on different cardinals.

Lemma 1.6. Let $\lambda$ be a regular uncountable cardinal, let $\nu<\lambda^{+}$, and let $g: \lambda \rightarrow \nu$ be a surjection. For $\beta \leq \nu$ fix $f_{\beta} \in \mathbf{f}_{\beta}^{\lambda}$. For each $\alpha<\lambda$, define a function $h_{\alpha}$ on $\alpha$ by letting $h_{\alpha}(i)=f_{g(i)}(\alpha)$ for all $i<\alpha$. Then there are club many $\alpha$ in $\lambda$ such that $h_{\alpha}$ is a surjection of $\alpha$ onto $f_{\nu}(\alpha)$.

Proof. Fix a club $C \subseteq \lambda$ such that for all $\alpha$ in $C, \alpha$ is a limit ordinal and $f_{\nu}(\alpha)=$ $\operatorname{ot}(g[\alpha])$. By Lemma 1.3, for each $\beta<\nu$ we can fix a club $C_{\beta} \subseteq \lambda$ such that

$$
\forall \alpha \in C_{\beta}\left(f_{\beta}(\alpha)=\operatorname{ot}(g[\alpha] \cap \beta)\right) .
$$

Let $D=C \cap \triangle\left\{C_{g(i)}: i<\lambda\right\}$. 
Let $\alpha$ be in $D$. We prove that $h_{\alpha}$ is a surjection of $\alpha$ onto $f_{\nu}(\alpha)$. First we show that $h_{\alpha}(i) \in f_{\nu}(\alpha)$ for all $i<\alpha$. If $i<\alpha$, then $\alpha \in C_{g(i)}$, and hence

$$
h_{\alpha}(i)=f_{g(i)}(\alpha)=\operatorname{ot}(g[\alpha] \cap g(i))<\operatorname{ot}(g[\alpha])=f_{\nu}(\alpha) .
$$

Let $\pi: g[\alpha] \rightarrow \operatorname{ot}(g[\alpha])$ be the transitive collapsing map of $g[\alpha]$. Then for all $i<\alpha, \pi(g(i))=\operatorname{ot}(g[\alpha] \cap g(i))$. To show that $h_{\alpha}$ is a surjection, consider $\gamma$ in $f_{\nu}(\alpha)=\operatorname{ot}(g[\alpha])$. Then $\pi^{-1}(\gamma) \in g[\alpha]$. Fix $i<\alpha$ such that $\pi^{-1}(\gamma)=g(i)$; then $\pi(g(i))=\gamma$. As $\alpha$ is in $C_{g(i)}$,

$$
h_{\alpha}(i)=f_{g(i)}(\alpha)=\operatorname{ot}(g[\alpha] \cap g(i))=\pi(g(i))=\gamma .
$$

Thus $h_{\alpha}$ is a surjection.

We will prove that a stronger version of the next proposition holds in $L$, in Lemma 2.3 of the next section.

Proposition 1.7. Let $\lambda$ be a regular uncountable cardinal. Let $\nu<\lambda^{+}$, and let $f_{\nu}$ be in $\mathbf{f}_{\nu}^{\lambda}$. Then there is a club $C \subseteq \lambda$ such that for all $\alpha$ in $C$, if $\alpha$ is regular and uncountable, then $f_{\nu}(\alpha)<\alpha^{+}$and $f_{\nu} \uparrow \alpha$ is in $\mathbf{f}_{f_{\nu}(\alpha)}^{\alpha}$.

Now we define the Mahlo hierarchy. The definition is by recursion.

Definition 1.8. Let $\lambda$ be a regular uncountable cardinal. Assume that for all regular uncountable cardinals $\alpha<\lambda$ and $\nu \leq \alpha^{+}$, we have defined what it means for $\alpha$ to be $\nu$-Mahlo. Let $\beta \leq \lambda^{+}$. We say that $\lambda$ is $\beta$-Mahlo if:

(1) $(\beta=0) \lambda$ is strongly inaccessible;

(2) $(\beta=\gamma+1)$ the set $\left\{\alpha \in \lambda: \alpha\right.$ is $f_{\gamma}^{\lambda}(\alpha)$-Mahlo $\}$ is stationary in $\lambda$, for some (any) $f_{\gamma}^{\lambda}$ in $\mathbf{f}_{\gamma}^{\lambda}$

(3) ( $\beta$ is a limit ordinal) $\lambda$ is $\gamma$-Mahlo for all $\gamma<\beta$.

Definition 1.9. Let $\lambda$ be a regular uncountable cardinal. Then $\lambda$ is greatly Mahlo if $\lambda$ is $\lambda^{+}$-Mahlo.

Suppose $\lambda$ is strongly inaccessible but not greatly Mahlo. Then the least $\nu$ such that $\lambda$ is not $\nu$-Mahlo must be a successor ordinal.

It can be easily proven by induction that if $\lambda$ is $\nu$-Mahlo, then $\lambda$ is $\beta$-Mahlo for all $\beta<\nu$.

\section{No Partial Squares Implies a Greatly Mahlo Cardinal}

We prove that for a regular uncountable cardinal $\kappa$, if no stationary subset of $\kappa^{+} \cap \operatorname{cof}(\kappa)$ carries a partial square, then $\kappa^{+}$is greatly Mahlo in $L$.

Theorem 2.1. Let $\kappa$ be a regular uncountable cardinal, and let $\lambda=\kappa^{+}$. Assume that $\lambda$ is not greatly Mahlo in L. Then there is a stationary subset of $\kappa^{+} \cap \operatorname{cof}(\kappa)$ which carries a partial square.

This theorem can be thought of as an extension of Jensen's theorem that if $\kappa^{+}$ is not a Mahlo cardinal in $L$, then $\square_{\kappa}$ holds. We will use the following result.

Theorem 2.2 (Jensen [6]). Let $\kappa$ be a regular uncountable cardinal, and let $\lambda=\kappa^{+}$. Let $W \subseteq \lambda$ be a set satisfying that for every limit ordinal $\eta<\lambda$ with $\kappa \leq \eta<\lambda$, the set

$$
W \cap[\kappa \cdot \eta, \kappa \cdot(\eta+1))
$$


codes a well-ordering of $\kappa$ of order type $\eta$. Let

$$
X=\{\alpha \in \lambda: \kappa \cdot \alpha=\alpha \wedge L[W \cap \alpha] \models|\alpha| \leq \kappa\} .
$$

Then there exists a sequence $\left\langle C_{\alpha}: \alpha \in X \cap \lim (X)\right\rangle$ satisfying:

(1) $C_{\alpha}$ is in $L[W \cap \alpha]$;

(2) $C_{\alpha}$ is a club subset of $\alpha$;

(3) ot $\left(C_{\alpha}\right) \leq \kappa$;

(4) $C_{\alpha} \subseteq X$;

(5) if $\gamma$ is a limit point of $C_{\alpha}$, then $C_{\alpha} \cap \gamma=C_{\gamma}$.

Note that the existence of such a set $W$ in $V$ is an easy consequence of $\lambda$ being the successor of $\kappa$. Also note that for all $\alpha<\lambda$, if $\kappa \cdot \alpha=\alpha$ and

$$
L \models \alpha \text { is singular, }
$$

then

$$
L[W \cap \alpha] \models|\alpha| \leq \kappa .
$$

This is true because $L[W \cap \alpha] \models \alpha \leq \kappa^{+}$by the choice of $W$; so if $\alpha$ is singular in $L[W \cap \alpha]$, then $\alpha$ is less than $\kappa^{+}$in $L[W \cap \alpha]$.

Let us begin the proof of Theorem 2.1. Let $\kappa$ be a regular uncountable cardinal, and let $\lambda=\kappa^{+}$. Assume that $\lambda$ is not greatly Mahlo in $L$.

The proof splits into two separate cases. Let

$$
Y=\{\alpha \in \lambda \cap \operatorname{cof}(\kappa): L \models \alpha \text { is singular }\} .
$$

We consider first the case that $Y$ is a stationary subset of $\lambda$ in $V$.

Let $W \subseteq \lambda$ be a set as described in the assumptions of Theorem 2.2, and let

$$
X=\{\alpha \in \lambda: \kappa \cdot \alpha=\alpha \wedge L[W \cap \alpha] \models|\alpha| \leq \kappa\} .
$$

Let $D$ be the club set of $\alpha<\lambda$ such that $\kappa \cdot \alpha=\alpha$. Then $Y \cap D \subseteq X$, as noted above. By Theorem 2.2, fix a sequence $\left\langle C_{\alpha}: \alpha \in X \cap \lim (X)\right\rangle$ satisfying:

(1) $C_{\alpha}$ is a club subset of $\alpha$ of order type at most $\kappa$;

(2) $C_{\alpha} \subseteq X$;

(3) if $\gamma$ is a limit point of $C_{\alpha}$, then $C_{\alpha} \cap \gamma=C_{\gamma}$.

Consider the restriction of this sequence to $Y \cap D$ :

$$
\left\langle C_{\alpha}: \alpha \in Y \cap D\right\rangle \text {. }
$$

Then for all $\alpha$ in $Y \cap D$, since $\operatorname{cf}(\alpha)=\kappa, C_{\alpha}$ is a club subset of $\alpha$ of order type $\kappa$. If $\alpha$ and $\beta$ are in $Y \cap D$ and $\gamma$ is a limit point of $C_{\alpha}$ and $C_{\beta}$, then

$$
C_{\alpha} \cap \gamma=C_{\gamma}=C_{\beta} \cap \gamma
$$

Thus the sequence $\left\langle C_{\alpha}: \alpha \in Y \cap D\right\rangle$ is a partial square sequence on a stationary subset of $\kappa^{+} \cap \operatorname{cof}(\kappa)$, and we are done.

From now on we will assume that the set

$$
\{\alpha \in \lambda \cap \operatorname{cof}(\kappa): L \models \alpha \text { is singular }\}
$$

is non-stationary in $V$. Clearly then $\lambda$ is a strongly inaccessible cardinal in $L$, so the set of limit cardinals of $L$ below $\lambda$ is club in $\lambda$. Fix a club set $C_{0} \subseteq \lambda$ in $V$ such that

$$
\forall \alpha \in C_{0} \cap \operatorname{cof}(\kappa)(L \models \alpha \text { is strongly inaccessible). }
$$


Let us fix canonical functions in $L$. For each ordinal $\alpha \leq \lambda$ which is regular and uncountable in $L$, and each ordinal $\delta<\left(\alpha^{+}\right)^{L}$, let $g_{\delta}^{\alpha}$ be the $L$-least surjection of $\alpha$ onto $\delta$. Define $f_{\delta}^{\alpha}: \alpha \rightarrow \alpha$ by

$$
f_{\delta}^{\alpha}(\gamma)=\operatorname{ot}\left(g_{\delta}^{\alpha}[\gamma]\right)
$$

Then $L$ models that $f_{\delta}^{\alpha}$ is a canonical function on $\alpha$ of rank $\delta$. Note that for each $\gamma$ in $\alpha, f_{\delta}^{\alpha}(\gamma)=\operatorname{ot}\left(g_{\delta}^{\alpha}[\gamma]\right)<\left(\gamma^{+}\right)^{L}$.

Lemma 2.3. Let $\beta<\left(\lambda^{+}\right)^{L}$. Then there is a club $E \subseteq \lambda$ in $L$ such that for all $\alpha$ in $E$, if $\alpha$ is regular and uncountable in $L$, then

$$
f_{\beta}^{\lambda}\left\lceil\alpha=f_{f_{\beta}^{\lambda}(\alpha)}^{\alpha} .\right.
$$

Proof. Let $N$ be the structure $L_{\left(\lambda^{+}\right)^{L}}$. For each $\alpha<\lambda$, let

$$
N_{\alpha}=S k^{N}(\alpha \cup\{\beta\}) \text {. }
$$

Let $E$ be the club set of $\alpha<\lambda$ such that $N_{\alpha} \cap \lambda=\alpha$.

Suppose $\alpha$ is in $E$, and $\alpha$ is regular and uncountable in $L$. By the Condensation Lemma, let $\pi: N_{\alpha} \rightarrow L_{\rho}$ be the transitive collapsing map, for some ordinal $\rho$. Since $N_{\alpha} \cap \lambda=\alpha, \pi(\lambda)=\alpha$.

We claim that $\pi(\beta)=f_{\beta}^{\lambda}(\alpha)$. Since $N=|\beta| \leq \lambda$, by the $L$-minimality of $g_{\beta}^{\lambda}$, this function is in $N_{\alpha}$. It follows by elementarity that

$$
N_{\alpha} \cap \beta=g_{\beta}^{\lambda}\left[N_{\alpha} \cap \lambda\right]=g_{\beta}^{\lambda}[\alpha] .
$$

Hence

$$
\pi(\beta)=\left\{\pi(\xi): \xi \in N_{\alpha} \cap \beta\right\}=\left\{\pi\left(g_{\beta}^{\lambda}(i)\right): i<\alpha\right\} .
$$

Since $\pi$ is an isomorphism, this ordinal is the order type of the set $\left\{g_{\beta}^{\lambda}(i): i<\alpha\right\}$, which is equal to ot $\left(g_{\beta}^{\lambda}[\alpha]\right)=f_{\beta}^{\lambda}(\alpha)$.

By elementarity, $\pi\left(g_{\beta}^{\lambda}\right)$ is the $L$-least surjection of $\pi(\lambda)=\alpha$ onto $\pi(\beta)=f_{\beta}^{\lambda}(\alpha)$. Therefore $\pi\left(g_{\beta}^{\lambda}\right)=g_{f_{\beta}^{\lambda}(\alpha)}^{\alpha}$. It follows that $\pi\left(f_{\beta}^{\lambda}\right)=f_{f_{\beta}^{\lambda}(\alpha)}^{\alpha}$. But $f_{\beta}^{\lambda} \cap N_{\alpha}=f_{\beta}^{\lambda} \uparrow \alpha$, and $\pi$ is the identity on this set. So $\pi\left(f_{\beta}^{\lambda}\right)=f_{\beta}^{\lambda}\lceil\alpha$.

Lemma 2.4. Let $\beta<\left(\lambda^{+}\right)^{L}$. Let $k: \lambda \rightarrow \lambda$ be a function. Suppose $A \subseteq \lambda$ is a set which is stationary in $V$ such that

$$
\forall \alpha \in A\left(k(\alpha)<f_{\beta}^{\lambda}(\alpha)\right)
$$

Then there is $\zeta<\beta$ and a set $B \subseteq A$ which is stationary in $V$ such that

$$
\forall \alpha \in B\left(k(\alpha)=f_{\zeta}^{\lambda}(\alpha)\right)
$$

Proof. For each $\alpha<\lambda$, let $h_{\alpha}: \alpha \rightarrow O n$ be the function

$$
h_{\alpha}(i)=f_{g_{\beta}^{\lambda}(i)}^{\lambda}(\alpha) \text {. }
$$

By Lemma 1.6, there is a club $E \subseteq \lambda$ in $L$ such that for all $\alpha$ in $E, h_{\alpha}$ is a surjection of $\alpha$ onto $f_{\beta}^{\lambda}(\alpha)$. Define a regressive function $m: A \cap E \rightarrow \lambda$ as follows. Let $\alpha$ be in $A \cap E$. Then $k(\alpha)<f_{\beta}^{\lambda}(\alpha)$. Since $h_{\alpha}$ is a surjection of $\alpha$ onto $f_{\beta}^{\lambda}(\alpha)$, let $m(\alpha)$ be the least ordinal less than $\alpha$ such that $h_{\alpha}(m(\alpha))=k(\alpha)$. By Fodor's Lemma, fix $i<\lambda$ and a set $B \subseteq A \cap C$ which is stationary in $V$ such that for all $\alpha$ in $B$, $m(\alpha)=i$. Let $\zeta=g_{\beta}^{\lambda}(i)$. Then for all $\alpha$ in $B$,

$$
k(\alpha)=h_{\alpha}(m(\alpha))=h_{\alpha}(i)=f_{g_{\beta}^{\lambda}(i)}^{\lambda}(\alpha)=f_{\zeta}^{\lambda}(\alpha) .
$$


Since $\lambda$ is not greatly Mahlo in $L$, let $\delta<\left(\lambda^{+}\right)^{L}$ be the least ordinal such that $\lambda$ is not $\delta+1$-Mahlo in $L$. Then the set

$$
\left\{\alpha \in \lambda: L \models \alpha \text { is } f_{\delta}^{\lambda}(\alpha) \text {-Mahlo }\right\}
$$

is non-stationary in $L$. Let $C_{1}$ be a club subset of $\lambda$ in $L$ such that

$$
\forall \alpha \in C_{1}\left(L \models \alpha \text { is not } f_{\delta}^{\lambda}(\alpha) \text {-Mahlo }\right) \text {. }
$$

Let $C=C_{0} \cap C_{1}$. Then for all $\alpha$ in $C \cap \operatorname{cof}(\kappa), \alpha$ is strongly inaccessible in $L$, but not $f_{\delta}^{\lambda}(\alpha)$-Mahlo in $L$.

For each $\alpha$ in $C \cap \operatorname{cof}(\kappa)$, let $\delta(\alpha)<\left(\alpha^{+}\right)^{L}$ be the least ordinal such that $\alpha$ not $\delta(\alpha)+1$-Mahlo in $L$. Then clearly

$$
\forall \alpha \in C \cap \operatorname{cof}(\kappa)\left(\delta(\alpha)<f_{\delta}^{\lambda}(\alpha)\right) .
$$

By Lemma 2.4, there is a set $A \subseteq C \cap \operatorname{cof}(\kappa)$ which is stationary in $V$ and an ordinal $\zeta<\delta$ such that

For each $\beta<\delta$, define

$$
\forall \alpha \in A\left(\delta(\alpha)=f_{\zeta}^{\lambda}(\alpha)\right)
$$

$$
A_{\beta}=\left\{\alpha \in C \cap \operatorname{cof}(\kappa): \delta(\alpha)=f_{\beta}^{\lambda}(\alpha)\right\} .
$$

We just noted that for some $\zeta<\delta, A_{\zeta}$ is stationary in $V$. Let $\beta$ be the least ordinal less than $\delta$ such that $A_{\beta}$ is stationary. By Lemma 2.3, fix a club set $E_{\beta} \subseteq \lambda$ in $L$ such that for all $\alpha$ in $E_{\beta}$, if $\alpha$ is regular and uncountable in $L$, then $f_{\beta}^{\lambda}\left\lceil\alpha=f_{f_{\beta}^{\lambda}(\alpha)}^{\alpha}\right.$.

Lemma 2.5. There exists a sequence $\left\langle d_{\alpha}: \alpha \in A_{\beta} \cap E_{\beta}\right\rangle$ satisfying:

(1) $d_{\alpha}$ is a club subset of $\alpha$;

(2) $\forall \gamma \in d_{\alpha}\left(L \models \gamma\right.$ is not $f_{\beta}^{\lambda}(\gamma)-$ Mahlo $)$;

(3) if $\gamma$ is a limit point of both $d_{\alpha_{1}}$ and $d_{\alpha_{2}}$, then $d_{\alpha_{1}} \cap \gamma=d_{\alpha_{2}} \cap \gamma$.

Proof. For each limit ordinal $\alpha<\lambda$, let $b_{\alpha}$ be the $L$-least closed and unbounded subset of $\alpha$ satisfying:

$$
L \models \forall \gamma \in b_{\alpha}\left(\gamma \text { is not } f_{\beta}^{\lambda}(\gamma) \text {-Mahlo }\right),
$$

if such a set exists. Otherwise let $b_{\alpha}$ be the empty set. Also let $\varphi_{\alpha}$ be the least ordinal larger than $\alpha$ and $f_{\beta}^{\lambda}(\alpha)$ satisfying:

(1) $L_{\varphi_{\alpha}}=Z F C^{-}$;

(2) $L_{\varphi_{\alpha}}=\left|f_{\beta}^{\lambda}(\alpha)\right| \leq \alpha$

(3) $b_{\alpha} \in L_{\varphi_{\alpha}}$.

Note that $\varphi_{\alpha}<\left(\alpha^{+}\right)^{L}$.

Fix $\alpha$ in $A_{\beta} \cap E_{\beta}$, and we will define $d_{\alpha}$. Since $\delta(\alpha)=f_{\beta}^{\lambda}(\alpha), \alpha$ is $f_{\beta}^{\lambda}(\alpha)$-Mahlo in $L$, but not $f_{\beta}^{\lambda}(\alpha)+1$-Mahlo in $L$. Therefore the set

$$
\left\{\gamma \in \alpha: L \models \gamma \text { is } f_{f_{\beta}^{\lambda}(\alpha)}^{\alpha}(\gamma) \text {-Mahlo }\right\}
$$

is non-stationary in L. But $f_{f_{\beta}^{\lambda}(\alpha)}^{\alpha}=f_{\beta}^{\lambda}\lceil\alpha$. So the set

$$
\left\{\gamma \in \alpha: L \models \gamma \text { is } f_{\beta}^{\lambda}(\gamma) \text {-Mahlo }\right\}
$$

is non-stationary in $L$. It follows that $b_{\alpha}$ is a club subset of $\alpha$ which is disjoint from this set. 
Recall that $g_{f_{\beta}^{\lambda}(\alpha)}^{\alpha}$ is the $L$-least surjection of $\alpha$ onto $f_{\beta}^{\lambda}(\alpha)$. By (2) in the definition of $\varphi_{\alpha}$, it follows that $g_{f_{\beta}^{\lambda}(\alpha)}^{\alpha}$ is in $L_{\varphi_{\alpha}}$. Therefore by $(1), f_{f_{\beta}^{\lambda}(\alpha)}^{\alpha}$ is in $L_{\varphi_{\alpha}}$, since this function is defined in an absolute way in $Z F C^{-}$from $g_{f_{\beta}^{\lambda}(\alpha)}^{\alpha}$. But $f_{f_{\beta}^{\lambda}(\alpha)}^{\alpha}=f_{\beta}^{\lambda}\left\lceil\alpha\right.$. So $f_{\beta}^{\lambda}\left\lceil\alpha\right.$ is in $L_{\varphi_{\alpha}}$.

Since $\alpha$ is strongly inaccessible in $L, L_{\varphi_{\alpha}}$ correctly computes the $L$-degree of Mahloness of cardinals below $\alpha$. Therefore $L_{\varphi_{\alpha}}$ models that $b_{\alpha}$ is the $L$-least club subset of $\alpha$ such that

$$
\forall \gamma \in b_{\alpha}\left(\gamma \text { is not } f_{\beta}^{\lambda}(\gamma) \text {-Mahlo }\right)
$$

For each $\gamma<\alpha$, let $N_{\gamma}$ be the Skolem hull

$$
S k^{L_{\varphi} \alpha}\left(\gamma \cup\left\{f_{\beta}^{\lambda}(\alpha)\right\}\right) \text {. }
$$

Since $\alpha$ is definable in $L_{\varphi_{\alpha}}$ as the largest cardinal, $\alpha$ is in $N_{\gamma}$. Also since $f_{\beta}^{\lambda} \uparrow \alpha$ and $b_{\alpha}$ are definable in $L_{\varphi_{\alpha}}$ from $f_{\beta}^{\lambda}(\alpha)$, they are in $N_{\gamma}$.

Now define

$$
d_{\alpha}=\left\{\gamma \in \alpha: N_{\gamma} \cap \alpha=\gamma\right\} .
$$

Clearly $d_{\alpha}$ is a club subset of $\alpha$ in $L$.

Consider $\gamma$ in $d_{\alpha}$. Since $b_{\alpha} \in N_{\gamma}, N_{\gamma} \cap \alpha=\gamma \in b_{\alpha}$ by elementarity. It follows that $\gamma$ is not $f_{\beta}^{\lambda}(\gamma)$-Mahlo in $L$. As the cardinal successor function below $\alpha$ is definable in $L_{\varphi_{\alpha}}, \gamma$ is a strong limit cardinal in $L$.

Since $N_{\gamma} \prec L_{\varphi_{\alpha}}$, by the Condensation Lemma the transitive collapse of $N_{\gamma}$ is equal to $L_{\psi}$ for some ordinal $\psi$. Let

$$
\pi_{\gamma}: N_{\gamma} \rightarrow L_{\psi}
$$

be the transitive collapsing map of $N_{\gamma}$. As $N_{\gamma} \cap \alpha=\gamma$,

$$
\pi_{\gamma}(\alpha)=\gamma \text {. }
$$

We claim that

$$
\pi_{\gamma}\left(f_{\beta}^{\lambda}(\alpha)\right)=f_{\beta}^{\lambda}(\gamma) .
$$

Since $f_{\beta}^{\lambda}\left\lceil\alpha=f_{f_{\beta}^{\lambda}(\alpha)}^{\alpha}\right.$, it suffices to show that

$$
\pi_{\gamma}\left(f_{\beta}^{\lambda}(\alpha)\right)=f_{f_{\beta}^{\lambda}(\alpha)}^{\alpha}(\gamma) .
$$

As $g_{f_{\beta}^{\lambda}(\alpha)}^{\alpha}$ is in $N_{\gamma}$ by elementarity, and $g_{f_{\beta}^{\lambda}(\alpha)}^{\alpha}: \alpha \rightarrow f_{\beta}^{\lambda}(\alpha)$ is a surjection,

$$
N_{\gamma} \cap f_{\beta}^{\lambda}(\alpha)=g_{f_{\beta}^{\lambda}(\alpha)}^{\alpha}\left[N_{\gamma} \cap \alpha\right]=g_{f_{\beta}^{\lambda}(\alpha)}^{\alpha}[\gamma] .
$$

Hence

$$
\pi_{\gamma}\left(f_{\beta}^{\lambda}(\alpha)\right)=\left\{\pi_{\gamma}(\xi): \xi \in N_{\gamma} \cap f_{\beta}^{\lambda}(\alpha)\right\}=\left\{\pi_{\gamma}\left(g_{f_{\beta}^{\lambda}(\alpha)}^{\alpha}(i)\right): i<\gamma\right\} .
$$

But $\pi_{\gamma}$ is order-preserving, so this ordinal is the order type of the set $g_{f_{\beta}^{\lambda}(\alpha)}^{\alpha}[\gamma]$, which equals $f_{f_{\beta}^{\lambda}(\alpha)}^{\alpha}(\gamma)$.

Now $N_{\gamma} \cap\left(f_{\beta}^{\lambda}\lceil\alpha)=f_{\beta}^{\lambda}\left\lceil\gamma\right.\right.$, and $\pi_{\gamma}$ is the identity on this set. So

$$
\pi_{\gamma}\left(f_{\beta}^{\lambda}\lceil\alpha)=f_{\beta}^{\lambda}\lceil\gamma .\right.
$$

By elementarity, it follows that $L_{\psi}$ models that $\pi_{\gamma}\left(b_{\alpha}\right)$ is the $L$-least closed and unbounded subset of $\gamma$ such that for all $\xi$ in $\pi_{\gamma}\left(b_{\alpha}\right), \xi$ is not $f_{\beta}^{\lambda}(\xi)$-Mahlo. Since 
$\gamma$ is a strong limit cardinal, $L_{\psi}$ correctly computes the $L$-degree of Mahloness of cardinals below $\gamma$. It follows that

$$
\pi_{\gamma}\left(b_{\alpha}\right)=b_{\gamma}
$$

To summarize, we have proven that $\pi_{\gamma}(\alpha)=\gamma, \pi_{\gamma}\left(f_{\beta}^{\lambda}(\alpha)\right)=f_{\beta}^{\lambda}(\gamma)$, and $\pi_{\gamma}\left(b_{\alpha}\right)=$ $b_{\gamma}$. Also, $\psi$ is the least ordinal larger than $\gamma$ and $f_{\beta}^{\lambda}(\gamma)$ satisfying:

(1) $L_{\psi} \models Z F C^{-}$;

(2) $L_{\psi} \models\left|f_{\beta}^{\lambda}(\gamma)\right| \leq \gamma$;

(3) $b_{\gamma} \in L_{\psi}$.

By elementarity, clearly $L_{\psi}$ satisfies these properties. If $\chi<\psi$ also satisfies these properties, then $\pi^{-1}\left(L_{\chi}\right)=L_{\pi^{-1}(\chi)}$ would contradict the minimality of $\varphi_{\alpha}$. It follows that $\psi=\varphi_{\gamma}$.

Since $N_{\gamma} \prec L_{\varphi_{\alpha}}$, for all $\xi<\gamma, N_{\xi}=S k^{N_{\gamma}}\left(\xi \cup\left\{f_{\beta}^{\lambda}(\alpha)\right\}\right)$. Since $\pi_{\gamma}: N_{\gamma} \rightarrow L_{\varphi_{\gamma}}$ is an isomorphism, it follows that

We now claim that for all $\xi<\gamma$,

$$
\pi_{\gamma}\left[N_{\xi}\right]=S k^{L_{\varphi}}\left(\xi \cup\left\{f_{\beta}^{\lambda}(\gamma)\right\}\right) ;
$$

$$
\xi \in d_{\alpha} \Longleftrightarrow S k^{L_{\varphi \gamma}}\left(\xi \cup\left\{f_{\beta}^{\lambda}(\gamma)\right\}\right) \cap \gamma=\xi ;
$$

in other words,

$$
N_{\xi} \cap \alpha=\xi \Longleftrightarrow \pi_{\gamma}\left[N_{\xi}\right] \cap \gamma=\xi
$$

Suppose $N_{\xi} \cap \alpha=\xi$. Since $\xi \subseteq N_{\xi}$ and $\pi_{\gamma}\left\lceil\gamma\right.$ is the identity, $\xi \subseteq \pi_{\gamma}\left[N_{\xi}\right] \cap \gamma$. Let $\rho$ be in $\pi_{\gamma}\left[N_{\xi}\right] \cap \gamma$. Then $\rho=\pi_{\gamma}\left(\rho^{\prime}\right)$ for some $\rho^{\prime} \in N_{\xi}$. Then $\pi_{\gamma}\left(\rho^{\prime}\right)=\rho<\gamma=\pi_{\gamma}(\alpha)$, so $\rho^{\prime}<\alpha$. Therefore $\rho^{\prime} \in N_{\xi} \cap \alpha=\xi$. So $\rho^{\prime} \in \xi$. But then $\rho=\pi_{\gamma}\left(\rho^{\prime}\right)=\rho^{\prime}$, so $\rho \in \xi$. Conversely, assume $\pi_{\gamma}\left[N_{\xi}\right] \cap \gamma=\xi$. Clearly $\xi \subseteq N_{\xi} \cap \alpha$. Let $\rho$ be in $N_{\xi} \cap \alpha$. Then $\pi_{\gamma}(\rho) \in \pi_{\gamma}(\alpha)=\gamma$, so $\pi_{\gamma}(\rho) \in \pi_{\gamma}\left[N_{\xi}\right] \cap \gamma=\xi=\pi_{\gamma}(\xi)$. So $\rho \in \xi$.

We have proven that for all $\alpha$ in $A_{\beta} \cap E_{\beta}$, for any $\gamma$ in $d_{\alpha}$,

$$
d_{\alpha} \cap \gamma=\left\{\xi \in \gamma: S k^{L_{\varphi_{\gamma}}}\left(\xi \cup\left\{f_{\beta}^{\lambda}(\gamma)\right\}\right) \cap \gamma=\xi\right\} .
$$

But the set on the right side is independent of $\alpha$. It follows that if $\alpha_{1}$ and $\alpha_{2}$ are in $A_{\beta} \cap E_{\beta}$, then for any $\gamma$ which is a limit point of $d_{\alpha_{1}}$ and $d_{\alpha_{2}}, d_{\alpha_{1}} \cap \gamma=d_{\alpha_{2}} \cap \gamma$.

Suppose that there exists a club set $F \subseteq \lambda$ such that for each $\alpha$ in the stationary set $A_{\beta} \cap E_{\beta} \cap \lim (F)$,

$$
\operatorname{ot}\left(d_{\alpha} \cap F\right)=\kappa .
$$

Then letting $c_{\alpha}=d_{\alpha} \cap F$ for all $\alpha$ in $A_{\beta} \cap E_{\beta} \cap \lim (F)$, clearly the sequence

$$
\left\langle c_{\alpha}: \alpha \in A_{\beta} \cap E_{\beta} \cap \lim (F)\right\rangle
$$

is a partial square sequence on a stationary subset of $\lambda \cap \operatorname{cof}(\kappa)$. So it suffices to show that there exists such a set $F$.

Suppose for a contradiction that for every club set $F \subseteq \lambda$, there is an ordinal $\alpha$ in $A_{\beta} \cap E_{\beta} \cap \lim (F)$ such that $d_{\alpha} \cap F$ has order type different from $\kappa$. Let

$$
B=\left\{\gamma \in \lambda \cap \operatorname{cof}(\kappa): \exists \alpha \in A_{\beta} \cap E_{\beta}\left(\gamma \in d_{\alpha}\right)\right\} .
$$

We claim that $B$ is stationary in $\lambda$.

To prove that $B$ is stationary, let $F \subseteq \lambda$ be a club. Then by assumption, there is some $\alpha$ in $A_{\beta} \cap E_{\beta} \cap \lim (F)$ such that $d_{\alpha} \cap F$ has order type different from $\kappa$. Now $d_{\alpha}$ and $F \cap \alpha$ are both club subsets of $\alpha$, and $\alpha$ has cofinality $\kappa$. So $d_{\alpha} \cap F$ is club in $\alpha$, and thus has order type at least $\kappa$. Therefore the order type of $d_{\alpha} \cap F$ is 
greater than $\kappa$. Let $\gamma$ be the $\kappa$-th element of $d_{\alpha} \cap F$. Then $\gamma$ has cofinality $\kappa$ and is in $d_{\alpha}$, and $\alpha$ is in $A_{\beta} \cap E_{\beta}$. So $\gamma$ is in $B$, and also $\gamma$ is in $F$.

We claim that for all $\gamma$ in $B \cap C, \delta(\gamma)<f_{\beta}^{\lambda}(\gamma)$. Consider $\gamma$ in $B \cap C$. Fix $\alpha$ in $A_{\beta} \cap E_{\beta}$ such that $\gamma \in d_{\alpha}$. Then by the choice of $d_{\alpha}, \gamma$ is not $f_{\beta}^{\lambda}(\gamma)$-Mahlo in $L$. But $\gamma$ is $\delta(\gamma)$-Mahlo in $L$. So $\delta(\gamma)<f_{\beta}^{\lambda}(\gamma)$.

Now we will get a contradiction. By Lemma 2.4, there is a set $B^{\prime} \subseteq B \cap C$ which is stationary in $V$ and an ordinal $\zeta<\beta$ such that

$$
\forall \gamma \in B^{\prime}\left(\delta(\gamma)=f_{\zeta}^{\lambda}(\gamma)\right)
$$

So $B^{\prime} \subseteq A_{\zeta}$, and therefore $A_{\zeta}$ is stationary. But $\zeta<\beta$, and $\beta$ is the minimal ordinal such that $A_{\beta}$ is stationary.

\section{Elementary Substructures and the Lévy Collapse}

We now turn towards proving the other direction of the equiconsistency result. We will start with a model in which $\kappa$ is a regular uncountable cardinal and $\lambda>\kappa$ is greatly Mahlo, and then produce a model by forcing in which $\lambda=\kappa^{+}$and there is no stationary subset of $\kappa^{+} \cap \operatorname{cof}(\kappa)$ which carries a partial square. The collapse of $\lambda$ to become $\kappa^{+}$will be achieved by using the Lévy collapse $\operatorname{CoLL}(\kappa,<\lambda)$. After forcing with the Lévy collapse, we will iterate forcing to destroy the stationarity of any subset of $\kappa^{+} \cap \operatorname{cof}(\kappa)$ which carries a partial square.

In this section we provide some preliminary results which will be used in the forcing proof, which concern elementary substructures and their interaction with the Lévy collapse.

For a set $N$ and a regular cardinal $\lambda$, we let $\lambda_{N}$ denote $N \cap \lambda$. As the notation suggests, we are interested in the case that $\lambda_{N}$ is an ordinal less than $\lambda$.

Notation 3.1. Let $\lambda$ be a strongly inaccessible cardinal. Define $S_{\lambda}$ as the set of $N$ in $P_{\lambda}\left(H\left(\lambda^{+}\right)\right)$satisfying:
(1) $N \prec H\left(\lambda^{+}\right)$;
(2) $\lambda_{N}$ is strongly inaccessible;
(3) $|N|=\lambda_{N}$;
(4) $N^{<\lambda_{N}} \subseteq N$.

Notation 3.2. Let $\lambda$ be a strongly inaccessible cardinal, and let $\nu<\lambda^{+}$. Define $S_{\nu}^{\lambda}$ as the set of $N$ in $S_{\lambda}$ such that there exists a canonical function $f_{\nu}$ on $\lambda$ of rank $\nu$ in $N$ such that $\lambda_{N}$ is $f_{\nu}\left(\lambda_{N}\right)$-Mahlo.

Lemma 3.3. Let $\lambda$ be a strongly inaccessible cardinal, and let $\nu<\lambda^{+}$. Suppose $\lambda$ is $\nu+1$-Mahlo. Then $S_{\nu}^{\lambda}$ is stationary in $P_{\lambda}\left(H\left(\lambda^{+}\right)\right)$.

Proof. Let $F: H\left(\lambda^{+}\right)^{<\omega} \rightarrow H\left(\lambda^{+}\right)$be a function. Build a sequence $\left\langle N_{i}: i<\lambda\right\rangle$ by induction satisfying:

(1) $N_{i} \prec\left(H\left(\lambda^{+}\right), \in, \nu, F\right)$;

(2) $\left|N_{i}\right|<\lambda$;

(3) $P\left(N_{i}\right) \subseteq N_{i+1}$

(4) if $\delta<\lambda$ is a limit ordinal, then $N_{\delta}=\bigcup\left\{N_{i}: i<\delta\right\}$.

Let $C$ be the club set of $\alpha<\lambda$ such that $N_{\alpha} \cap \lambda=\alpha=\left|N_{\alpha}\right|$.

By elementarity, fix a surjective function $g_{\nu}: \lambda \rightarrow \nu$ in $N_{0}$. Let $f_{\nu}: \lambda \rightarrow \lambda$ be the function $f_{\nu}(\alpha)=\operatorname{ot}\left(g_{\nu}[\alpha]\right)$. Then $f_{\nu}$ is in $N_{0}$ by elementarity, and $f_{\nu}$ is a 
canonical function on $\lambda$ of rank $\nu$. Since $\lambda$ is $\nu+1$-Mahlo, fix $\alpha$ in $C$ such that $\alpha$ is $f_{\nu}(\alpha)$-Mahlo. Then $N_{\alpha}$ is in $S_{\nu}^{\lambda}$ and is closed under $F$.

The next definition is standard, although it is usually considered in the case when $\lambda$ is strongly inaccessible. We will also use this idea when $\lambda$ is a successor cardinal; see Lemma 3.7 below for the context.

Definition 3.4. Let $\lambda$ be a regular uncountable cardinal. A set $N$ is a $\lambda$-model if:

(1) $(N, \in)$ is a model of $Z F C^{-}$;

(2) $N$ is transitive;

(3) $|N|=\lambda$;

(4) $\lambda \in N$;

(5) $N^{<\lambda} \subseteq N$.

Lemma 3.5. Let $\lambda$ be strongly inaccessible, and suppose $N$ is in $S_{\lambda}$. Then $\bar{N}$, the transitive collapse of $N$, is a $\lambda_{N}$-model.

Proof. Let $\pi: N \rightarrow \bar{N}$ be the transitive collapsing map. Since $N \prec H\left(\lambda^{+}\right), N$ is a model of $Z F C^{-}$. So $\bar{N}$ is a model of $Z F C^{-}$, as $\pi$ is an isomorphism. Obviously $\bar{N}$ is transitive, by the definition of the transitive collapse. Also $|\bar{N}|=|N|=\lambda_{N}$. As $N \cap \lambda=\lambda_{N}, \pi(\lambda)=\lambda_{N}$ is in $\bar{N}$. Finally, since $N^{<\lambda_{N}} \subseteq N$ and $N$ is isomorphic to $\bar{N}, \bar{N}^{<\lambda_{N}} \subseteq \bar{N}$.

Lemma 3.6. Let $\lambda$ be strongly inaccessible, and let $\nu<\lambda^{+}$. Let $N$ be in $S_{\lambda}$. Suppose $f_{\nu} \in N$ is a canonical function on $\lambda$ of rank $\nu$. Let $\pi: N \rightarrow \bar{N}$ be the transitive collapsing map. Then $\pi(\nu)=f_{\nu}\left(\lambda_{N}\right)$.

Proof. By elementarity, fix a surjection $g_{\nu}: \lambda \rightarrow \nu$ in $N$ and a club $C \subseteq \lambda$ in $N$ such that for all $\alpha$ in $C, f_{\nu}(\alpha)=\operatorname{ot}\left(g_{\nu}[\alpha]\right)$. Since $C \in N, \lambda_{N} \in C$, so $f_{\nu}\left(\lambda_{N}\right)=$ ot $\left(g_{\nu}\left[\lambda_{N}\right]\right)$. As $g_{\nu}$ is in $N$, clearly $N \cap \nu=g_{\nu}\left[\lambda_{N}\right]$ by elementarity. So $\pi(\nu)=\{\pi(\alpha)$ : $\alpha \in N \cap \nu\}=\left\{\pi\left(g_{\nu}(i)\right): i \in \lambda_{N}\right\}=\pi\left[g_{\nu}\left[\lambda_{N}\right]\right]$. Hence $\pi$ is an order preserving bijection between $\pi(\nu)$ and $g_{\nu}\left[\lambda_{N}\right]$, and therefore $\pi(\nu)=\operatorname{ot}\left(g_{\nu}\left[\lambda_{N}\right]\right)=f_{\nu}\left(\lambda_{N}\right)$.

Now we turn to the Lévy collapse. Suppose $\kappa$ is a regular uncountable cardinal, and $\lambda>\kappa$ is strongly inaccessible. The Lévy collapse $\operatorname{CoLL}(\kappa,<\lambda)$ is the forcing poset consisting of conditions $p$ which satisfy:

(1) $p: \kappa \times \lambda \rightarrow \lambda$ is a partial function;

(2) $|\operatorname{dom}(p)|<\kappa$;

(3) $p(\alpha, \beta)<\beta$ for all $\langle\alpha, \beta\rangle$ in $\operatorname{dom}(p)$.

Let $q \leq p$ if $q$ extends $p$ as a function.

We assume that the reader is familiar with the basic theory of the Lévy collapse; see [5] or [8] . For example, $\operatorname{Colv}(\kappa,<\lambda)$ is $\kappa$-closed and $\lambda$-c.c., and collapses $\lambda$ to become $\kappa^{+}$. If $\kappa<\bar{\lambda}<\lambda$ and $\bar{\lambda}$ is strongly inaccessible, then $\operatorname{CoLL}(\kappa,<\lambda)$ factors as

$$
\operatorname{CoLL}(\kappa,<\bar{\lambda}) \times \operatorname{CoLL}(\kappa,[\bar{\lambda}, \lambda)),
$$

where $\operatorname{CoLL}(\kappa,[\bar{\lambda}, \lambda))$ is the suborder of $\operatorname{CoLL}(\kappa,<\lambda)$ consisting of conditions $p$ such that $\operatorname{dom}(p) \subseteq \kappa \times[\bar{\lambda}, \lambda)$.

Note that if $N$ is a $\lambda$-model, then the Lévy collapse $\operatorname{CoLL}(\kappa,<\lambda)$ is a member of $N$. 
Lemma 3.7. Let $\kappa$ be a regular uncountable cardinal, and assume $\lambda>\kappa$ is strongly inaccessible. Let $N$ be a $\lambda$-model. Suppose $G$ is a generic filter on $\operatorname{CoLL}(\kappa,<\lambda)$. Then in $V[G], N[G]$ is a $\lambda$-model. Moreover, $N[G] \cap V=N$.

Proof. By basic facts of forcing, since $N$ is a transitive model of $Z F C^{-}$, the same is true of $N[G]$. Also $N$ and $N[G]$ have the same cardinality, which is $\lambda$, since $\lambda$ is preserved in $V[G]$. As $\lambda \in N$ and $N \subseteq N[G], \lambda \in N[G]$. It remains to show that $N[G]^{<\lambda} \subseteq N[G]$.

Since $\lambda=\kappa^{+}$in $V[G]$, it suffices to show that $N[G]^{\kappa} \subseteq N[G]$ in $V[G]$. Let $f: \kappa \rightarrow N[G]$ be a function. Define $g: \kappa \rightarrow N$ in $V[G]$ by choosing for each $i<\kappa$ a name $g(i)$ in $N$ such that $g(i)^{G}=f(i)$. Since $G \in N[G]$, it suffices to show that $g$ is in $N[G]$.

Fix a name $\dot{g}$ satisfying that $\dot{g}^{G}=g$ and $\operatorname{CoLL}(\kappa,<\lambda)$ forces that $\dot{g}$ is a function from $\kappa$ into names in $N$. For each $i<\kappa$, let $A_{i}$ be a maximal antichain contained in the dense set of conditions which decide $\dot{g}(i)$. Since $\operatorname{CoLL}(\kappa,<\lambda)$ is $\lambda$-c.c., $\left|A_{i}\right|<\lambda$. So $A_{i}$ is in $N$, because $N^{<\lambda} \subseteq N$. Therefore the sequence $\left\langle A_{i}: i<\kappa\right\rangle$ is in $N$.

Now define a name $\dot{h}$ in $N$ by letting $\langle p, \dot{a}\rangle$ be in $\dot{h}$ iff for some $i<\kappa, p \in A_{i}$, and $\dot{a}$ is the canonical name for a pair $\langle i, \check{x}\rangle$, where $p \Vdash \dot{g}(i)=\check{x}$. It is easy to check that $\dot{h}^{G}=g$, so $g$ is in $N[G]$ as desired.

The statement that $N[G] \cap V=N$ follows by a standard argument from the fact that $\operatorname{CoLL}(\kappa,<\lambda)$ is $\lambda$-c.c. If $\dot{a}$ is in $N$ and $\dot{a}^{G} \in V$, then there is a maximal antichain in $N$ consisting of conditions which either force that $\dot{a}$ is not in $V$, or otherwise decide the value of $\dot{a}$. Then $|A|<\lambda$, so $A \subseteq N$. Therefore every possible value for $\dot{a}$ is in $N$ by elementarity. In particular, $\dot{a}^{G} \in N$.

Lemma 3.8. Let $\kappa$ be a regular uncountable cardinal, and let $\lambda>\kappa$ be strongly inaccessible. Assume that $N$ is in $S_{\lambda}$ and $\kappa<\lambda_{N}$. Let $\pi: N \rightarrow \bar{N}$ be the transitive collapsing map of $N$. Suppose $G$ is a generic filter on $\operatorname{CoLL}(\kappa,<\lambda)$. Let $\sigma: N[G] \rightarrow$ $\overline{N[G]}$ be the transitive collapsing map of $N[G]$. Then

$$
\sigma \uparrow N=\pi
$$

and

$$
\overline{N[G]}=\bar{N}\left[G \uparrow \lambda_{N}\right] .
$$

In particular, $\overline{N[G]}$ is in $V\left[G \uparrow \lambda_{N}\right]$ and is a $\lambda_{N}$-model.

The first two assertions were proved in Lemma 6.4 of [7], and we will not repeat the proof here. For the last statement, $\bar{N}$ is a $\lambda_{N}$-model by Lemma 3.5, so $\bar{N}[G \uparrow$ $\left.\lambda_{N}\right]=\overline{N[G]}$ is a $\lambda_{N}$-model by Lemma 3.7 .

\section{Absoluteness of Partial Square Killing}

In this section we introduce the type of forcing iteration we will use, which we call a partial square killing forcing iteration. We need to give a general definition of this kind of iteration, rather than just constructing one iteration for the consistency proof. The reason is that we need an absoluteness result which states that if a $\lambda$ model believes that $\mathbb{P}$ is a partial square killing forcing iteration, then $\mathbb{P}$ really is such an iteration.

Let $\lambda$ be a regular uncountable cardinal. Define $X_{\lambda}$ by $x \in X_{\lambda} \Longleftrightarrow x$ is a closed, bounded, non-empty subset of $\lambda$. 
Define a relation $\leq_{\lambda}$ on $X_{\lambda}$ by

$$
q \leq_{\lambda} p \Longleftrightarrow q \cap(\max (p)+1)=p,
$$

that is, $q$ end-extends $p$.

For an ordinal $\nu$, define $X_{\lambda, \nu}$ by

$$
p \in X_{\lambda, \nu} \Longleftrightarrow p: \nu \rightarrow X_{\lambda} \text { is a partial function of size less than } \lambda \text {. }
$$

Define a relation $\leq_{\lambda, \nu}$ on $X_{\lambda, \nu}$ by

$$
q \leq_{\lambda, \nu} p \Longleftrightarrow \operatorname{dom}(p) \subseteq \operatorname{dom}(q) \wedge \forall \alpha \in \operatorname{dom}(p)\left(q(\alpha) \leq_{\lambda} p(\alpha)\right) .
$$

We also fix formulas in LST which describe $X_{\lambda}, \leq_{\lambda}, X_{\lambda, \nu}$, and $\leq_{\lambda, \nu}$. Let $\varphi_{0}(x, \lambda)$ be the formula of LST expressing that $x$ is a nonempty, closed, bounded subset of $\lambda$. Let $\psi_{0}(x, y, \lambda)$ be the formula of LST which asserts that $\varphi_{0}(x, \lambda)$ and $\varphi_{0}(y, \lambda)$ hold, and $y$ is an end-extension of $x$. Similarly fix formulas $\varphi_{1}(x, \lambda, \nu)$ and $\psi_{1}(x, y, \lambda, \nu)$ of LST to describe $X_{\lambda, \nu}$ and $\leq_{\lambda, \nu}$. It is easy to check that all these expressions are $\Delta_{0}$, except the part of $\varphi_{1}$ which asserts that $|x|<\lambda$, which is $\Sigma_{1}$.

Lemma 4.1. Let $\lambda$ be a regular uncountable cardinal, and let $N$ be a $\lambda$-model.

(1) $X_{\lambda} \subseteq N$;

(2) for all $x, x \in X_{\lambda}$ iff $N \models \varphi_{0}(x, \lambda)$;

(3) for all $x, y \in X_{\lambda}, y \leq_{\lambda} x$ iff $N \models \psi_{0}(x, y, \lambda)$.

Proof. (1) follows from the fact that $N^{<\lambda} \subseteq N$. (2) and (3) hold because $\varphi_{0}$ and $\psi_{0}$ are $\Delta_{0}$.

Lemma 4.2. Let $\lambda$ be a regular uncountable cardinal, and let $N$ be a $\lambda$-model. Let $\nu$ be an ordinal in $N$.

(1) $X_{\lambda, \nu} \subseteq N$

(2) for all $x, x \in X_{\lambda, \nu}$ iff $N=\varphi_{1}(x, \lambda, \nu)$;

(3) for all $x, y \in X_{\lambda, \nu}, y \leq_{\lambda, \nu} x$ iff $N \models \psi_{1}(x, y, \lambda, \nu)$.

Proof. (1) follows from the fact that $N^{<\lambda} \subseteq N$. (2) If $N=\varphi_{1}(x, \lambda, \nu)$, then since $\varphi_{1}$ is $\Sigma_{1}, x$ is in $X_{\lambda, \nu}$. If $x$ is in $X_{\lambda, \nu}$, then $x \in N$ by (1). Since $N^{<\lambda} \subseteq N$, $N \models|x|<\lambda$. The rest of $\varphi_{1}$ is $\Delta_{0}$, so $N \models \varphi_{1}(x, \lambda, \nu)$. (3) follows from (1) and (2) and the absoluteness of end-extension.

If $\mathbb{Q}$ is a suborder of $X_{\lambda, \nu}$ and $\zeta<\nu$, let

$$
\mathbb{Q} \uparrow \zeta=\{p \uparrow \zeta: p \in \mathbb{Q}\},
$$

ordered by $\leq_{\lambda, \zeta}$. It is easy to see that if $N$ is a $\lambda$-model, $\nu \in N$, and $\mathbb{Q}$ is a suborder of $X_{\lambda, \nu}$ in $N$, then for all $\zeta \in N \cap \nu, N$ computes $\mathbb{Q}\lceil\zeta$ correctly.

Lemma 4.3. Let $\lambda$ be a regular uncountable cardinal, and let $N$ be a $\lambda$-model. Let $x$ be a set in $N$, and let $\mathbb{P}$ be a forcing poset in $N$. Then $N$ models that $\dot{T}$ is a nice $\mathbb{P}$-name for a subset of $x$ iff $\dot{T}$ really is a nice $\mathbb{P}$-name for a subset of $x$.

Proof. The set $\dot{T}$ being a nice $\mathbb{P}$-name for a subset of $x$ can be expressed as follows:

(1) for all $a$ in $\dot{T}$, there is $p \in \mathbb{P}$ and $z \in x$ such that $a=\langle p, \check{z}\rangle$;

(2) for all $z$ in $x$ and $p, q$ in $\mathbb{P}$, if $\langle p, \check{z}\rangle$ and $\langle q, \check{z}\rangle$ are in $\dot{T}$, then $p$ and $q$ are incompatible.

The statement " $y=\check{z}$ " is absolute for transitive models of $Z F C^{-}$, and the formula above is $\Delta_{0}$ in this statement. 
Suppose $\mathbb{P}$ is a forcing poset, $\dot{a}_{1}, \ldots, \dot{a}_{n}$ are $\mathbb{P}$-names, and $\varphi\left(x_{1}, \ldots, x_{n}\right)$ is a formula which is absolute for transitive models of $Z F C^{-}$. Let $p \in \mathbb{P}$. Then the statement " $p \Vdash \varphi\left[\dot{a}_{1}, \ldots, \dot{a}_{n}\right]$ " is absolute between transitive models of $Z F C^{-}$.

Lemma 4.4. Suppose $\kappa$ is a regular uncountable cardinal, $\lambda=\kappa^{+}$, and $N$ is a $\lambda$-model. Let $\mathbb{P}$ be a forcing poset, and let $\dot{T}$ be a nice $\mathbb{P}$-name for a subset of $\lambda \cap \operatorname{cof}(\kappa)$. Suppose

$$
N \models(p \Vdash \dot{T} \text { carries a partial square }) .
$$

Then $p$ forces that $\dot{T}$ carries a partial square.

Proof. Fix a sequence $\left\langle\dot{c}_{\alpha}: \alpha<\lambda\right\rangle$ in $N$ such that $N$ models that $p$ forces:

(1) $\dot{c}_{\alpha}$ is non-empty iff $\alpha \in \dot{T}$;

(2) if $\alpha \in \dot{T}$, then $\dot{c}_{\alpha}$ is a club subset of $\alpha$ of order type $\kappa$;

(3) if $\gamma$ is a limit point of $\dot{c}_{\alpha}$ and $\dot{c}_{\beta}$, then $\dot{c}_{\alpha} \cap \gamma=\dot{c}_{\beta} \cap \gamma$.

Statements (1), (2), and (3) are absolute for transitive models of $Z F C^{-}$. So $p$ really does force (1), (2), and (3).

In the last lemma we are not assuming any cardinal preservation by the forcing poset $\mathbb{P}$. So in general it might not be clear what is meant by saying that $p$ forces that $\dot{T}$ carries a partial square. For clarity, what we mean is exactly what is described in the proof.

If $\mathbb{P}$ is a suborder of $X_{\lambda, \nu}$ and $\beta<\nu$, let

$$
\mathbb{P}_{\beta}=\mathbb{P} \cap X_{\lambda, \beta},
$$

considered as a suborder of $X_{\lambda, \beta}$. Note that if $N$ is a $\lambda$-model and $\mathbb{P} \in N$, then for all $\beta \in N \cap \nu, N$ computes $\mathbb{P}_{\beta}$ correctly.

Now we are ready to introduce the idea of a partial square killing forcing iteration.

Definition 4.5. Suppose $\kappa$ is a regular uncountable cardinal, $\lambda=\kappa^{+}$, and $\nu$ is an ordinal. We say that $\mathbb{P}$ is a partial square killing forcing iteration on $\lambda$ of length $\nu$ if there exists a sequence $\left\langle\dot{T}_{i}: i<\nu\right\rangle$ such that:

(1) $\mathbb{P}$ is a suborder of $X_{\lambda, \nu}$;

(2) the empty function is in $\mathbb{P}$;

(3) for all $\beta<\nu, \dot{T}_{\beta}$ is a nice $\mathbb{P}_{\beta}$-name for a subset of $\lambda \cap \operatorname{cof}(\kappa)$;

(4) for all $\beta<\nu, \mathbb{P}_{\beta}$ forces that $\dot{T}_{\beta}$ carries a partial square sequence;

(5) for all $p, p$ is in $\mathbb{P}$ iff ( $p$ is in $X_{\lambda, \nu}$ and for all $\alpha$ in $\operatorname{dom}(p), p\lceil\alpha \in \mathbb{P}$ and $p\left\lceil\alpha \Vdash_{\mathbb{P}_{\alpha}} p(\alpha) \cap \dot{T}_{\alpha}=\emptyset\right)$.

Fix a formula $\theta(\mathbb{P}, \lambda, \nu)$ in LST which asserts that $\mathbb{P}$ is a partial square killing forcing iteration on $\lambda$ of length $\nu$. The formula $\theta(\mathbb{P}, \lambda, \nu)$ is the conjunction of the statements (1)-(5) above, except that we replace any mention of $X_{\lambda, \nu}$ and $\leq_{\lambda, \nu}$ with the formulas $\varphi_{1}$ and $\psi_{1}$.

Proposition 4.6. Suppose $\kappa$ is a regular uncountable cardinal, $\lambda=\kappa^{+}$, and $\nu$ is an ordinal. Assume that $N$ is a $\lambda$-model and $\nu \in N$. If

$$
N \models \theta(\mathbb{P}, \lambda, \nu),
$$

then $\mathbb{P}$ is a partial square killing forcing iteration on $\lambda$ of length $\nu$. 
Proof. Fix in $N$ a sequence $\left\langle\dot{T}_{i}: i<\nu\right\rangle$ such that $N$ models (the modified versions using $\varphi_{1}$ and $\psi_{1}$ of) (1)-(5) of Definition 4.5. Lemma 4.2(2,3) implies that (1) holds for $\mathbb{P}$. (2) is immediate. For (3) and (4), let $\beta<\nu$. Then $N$ computes $\mathbb{P}_{\beta}$ correctly. Also note that since $N^{\kappa} \subseteq N$, the set $\lambda \cap \operatorname{cof}(\kappa)$ is computed correctly in $N$. Lemmas 4.3 and 4.4 now imply (3) and (4). (5) follows easily from Lemma 4.2 and the comments preceding Lemma 4.4 .

Note that if $\nu<\lambda^{+}$, then

$$
H\left(\lambda^{+}\right) \models \theta(\mathbb{P}, \lambda, \nu)
$$

iff $\mathbb{P}$ is a partial square killing forcing iteration on $\lambda$ of length $\nu$, because the properties described in Definition 4.5 are absolute between $H\left(\lambda^{+}\right)$and $V$.

\section{Properties of Partial Square Killing}

We will now discuss the basic properties of partial square killing forcing iterations. All of these properties are straightforward to prove, except for distributivity, which is handled in the next section using a large cardinal assumption. The proofs in this section will be brief or omitted, since they were already dealt with rigorously in [7]. In any case, the reader should have little difficulty filling in the details, if interested.

All of the properties developed in this section hold for any forcing iteration which kills subsets of $\lambda \cap \operatorname{cof}(\kappa)$, that is, for any $\mathbb{P}$ satisfying Definition $4.5(1,2,3,5)$.

When we say that a forcing poset $\mathbb{Q}$ is $\lambda$-distributive, we mean $<\lambda$-distributive, that is, any family of fewer than $\lambda$ many dense open subsets of $\mathbb{Q}$ has dense intersection. A set $H \subseteq \mathbb{Q}$ is said to be $N$-generic for $\mathbb{Q}$ if whenever $D \in N$ is a dense subset of $\mathbb{Q}$, then $H \cap D \cap N \neq \emptyset$.

Let $\kappa$ be a regular uncountable cardinal, and let $\lambda=\kappa^{+}$. Suppose $T$ is a subset of $\lambda \cap \operatorname{cof}(\kappa)$, not necessarily stationary. We let $\mathbb{P}(T)$ denote the forcing poset whose conditions are closed, bounded, non-empty subsets of $\lambda$ which are disjoint from $T$, ordered by end-extension. In other words,

$$
\forall p\left(p \in \mathbb{P}(T) \Longleftrightarrow\left(p \in X_{\lambda} \wedge p \cap T=\emptyset\right)\right)
$$

and

$$
\forall p, q \in \mathbb{P}(T)\left(q \leq_{\mathbb{P}(T)} p \Longleftrightarrow q \leq_{\lambda} p\right) .
$$

It is easy to show that if $p$ and $q$ are compatible in $\mathbb{P}(T)$, then either $q \leq \mathbb{P}(T) p$ or $p \leq_{\mathbb{P}(T)} q$. So every family of pairwise compatible conditions in $\mathbb{P}(T)$ is a chain. Also note that any chain $B \subseteq \mathbb{P}(T)$ of size less than $\kappa$ has a lower bound. For let $\alpha=\sup \{\max (p): p \in B\}$, and let $q=\bigcup B \cup\{\alpha\}$. Then either $\alpha$ belongs to some $p$ in $B$, which implies that $q=p$, or $\alpha$ has cofinality less than $\kappa$, and hence is not in $T$. In either case, $q$ is a condition and $q \leq_{\mathbb{P}(T)} p$ for all $p$ in $B$. In particular, $\mathbb{P}(T)$ is $\kappa$-closed. In general, however, $\mathbb{P}(T)$ might collapse $\lambda$.

For the rest of the section, fix a regular uncountable cardinal $\kappa$ and an ordinal $\nu$, and let $\lambda=\kappa^{+}$. Also fix $\mathbb{P}$ which satisfies Definition $4.5(1,2,3,5)$, witnessed by a sequence of names $\left\langle\dot{T}_{i}: i<\nu\right\rangle$.

Recall that for all $\beta<\nu$,

$$
\mathbb{P} \uparrow \beta=\{p\lceil\beta: p \in \mathbb{P}\}
$$

and

$$
\mathbb{P}_{\beta}=\mathbb{P} \cap X_{\lambda, \beta}
$$


The next two results are easy.

Lemma 5.1. For all $\beta<\nu, \mathbb{P}_{\beta}=\mathbb{P} \uparrow \beta$.

Proof. Definition $4.5(5)$ easily implies that $\mathbb{P}$ is closed under restrictions. So $\mathbb{P} \uparrow$ $\beta \subseteq \mathbb{P} \cap X_{\lambda, \beta}=\mathbb{P}_{\beta}$. Conversely, if $p$ is in $\mathbb{P} \cap X_{\lambda, \beta}$, then $p=p \uparrow \beta \in \mathbb{P} \uparrow \beta$.

Lemma 5.2. For all $\beta<\nu, \mathbb{P}_{\beta}$ is a partial square killing forcing iteration on $\lambda$ of length $\beta$.

Proof. It is straightforward to verify Definition 4.5 for $\mathbb{P}_{\beta}$. (1)-(4) are trivial. (5) follows easily using the fact that $\left(\mathbb{P}_{\beta}\right)_{\alpha}=\mathbb{P}_{\alpha}$.

The next lemma describes the relationship between $\mathbb{P}$ and its initial segments. The proof is straightforward, and we omit it. The least trivial statement is (2); this follows from Definition 4.5(5).

Lemma 5.3. Let $\beta<\nu$.

(1) $\mathbb{P}_{\beta} \subseteq \mathbb{P}$

(2) if $q$ is in $\mathbb{P}$ and $s \leq q \uparrow \beta$ in $\mathbb{P}_{\beta}$, then letting $t=s \cup q \uparrow[\beta, \alpha), t$ is in $\mathbb{P}$ and $t \leq s, q$ in $\mathbb{P}$

(3) the inclusion map $\mathbb{P}_{\beta} \rightarrow \mathbb{P}$ is a complete embedding;

(4) if $p$ and $q$ are in $\mathbb{P}$ and $q \leq p$ in $\mathbb{P}$, then $q \uparrow \beta \leq p \uparrow \beta$ in $\mathbb{P}_{\beta}$;

(5) if $u$ and $t$ are in $\mathbb{P}_{\beta}$, then $u \leq t$ in $\mathbb{P}_{\beta}$ iff $u \leq t$ in $\mathbb{P}$.

Lemma 5.4. Let $p$ be in $X_{\lambda, \nu}$ but not in $\mathbb{P}$. Then there is some $\alpha \in \operatorname{dom}(p)$ such that $p\left\lceil\alpha\right.$ is in $\mathbb{P}_{\alpha}$, but $p\left\lceil\alpha\right.$ does not force that $p(\alpha)$ is disjoint from $\dot{T}_{\alpha}$.

Proof. Let $\alpha$ be the least ordinal such that Definition 4.5(5) fails for $p$. By Definition 4.5(5), $p\lceil\alpha$ is in $\mathbb{P}$.

Lemma 5.5. Let $p$ and $q$ be incompatible conditions in $\mathbb{P}$. Then there is some $\beta$ in $\operatorname{dom}(p) \cap \operatorname{dom}(q)$ such that neither of $p(\beta)$ nor $q(\beta)$ is an initial segment of the other.

Proof. If the conclusion of the lemma fails, then it is easy to construct a lower bound of $p$ and $q$.

We make a comment about notation. Sometimes when we consider a function $p$ in $X_{\lambda, \nu}$ and an ordinal $\beta<\nu$, it will be convenient to write " $p(\beta)$ " without knowing whether or not $\beta$ is in the domain of $p$. In the case that it is not, $p(\beta)$ will denote the empty set.

Lemma 5.6. The forcing poset $\mathbb{P}$ is $\kappa$-closed. In fact, suppose $B$ is a directed subset of $\mathbb{P}$ of size less than $\kappa$. Define $q$ in $X_{\lambda, \nu}$ by letting

$$
\operatorname{dom}(q)=\bigcup\{\operatorname{dom}(p): p \in B\}
$$

and for all $\beta$ in $\operatorname{dom}(q)$,

$$
q(\beta)=\bigcup\{p(\beta): p \in B\} \cup\{\sup \{\max (p(\beta)): p \in B\}\} .
$$

Then $q$ is in $\mathbb{P}$ and $q \leq p$ for all $p$ in $B$.

Proof. If $\{p(\beta): p \in B\}$ has a largest set $s(\beta)$, then $q(\beta)=s(\beta)$. Otherwise the largest ordinal of $q(\beta)$ has cofinality less than $\kappa$, and therefore is forced to be not in $\dot{T}_{\beta}$. The lemma follows easily from these observations and Lemma 5.4. 
Lemma 5.7. Suppose $2^{\kappa}=\lambda$. If $\nu<\lambda^{+}$, then $|\mathbb{P}|<\lambda^{+}$. If $\nu \geq \lambda^{+}$, then $\mathbb{P}$ is $\lambda^{+}$-c.c.

Proof. A straightforward computation shows that if $2^{\kappa}=\lambda$, then $\left|X_{\lambda}\right|=\lambda$, and $\left|X_{\lambda, \nu}\right| \leq \lambda$ for all $\nu<\lambda^{+}$. Therefore $|\mathbb{P}| \leq \lambda$ if $\nu<\lambda^{+}$. In general, a straightforward argument using the $\Delta$-System Lemma and Lemma 5.5 shows that $\mathbb{P}$ is $\lambda^{+}$-c.c.

The next lemma describes the purpose of a partial square killing forcing iteration, namely, that it successively destroys the stationarity of sets which carry a partial square.

Lemma 5.8. Let $\beta<\nu$ and suppose that $\mathbb{P}_{\beta}$ is $\lambda$-distributive. Then $\mathbb{P}_{\beta+1}$ is isomorphic to a dense subset of $\mathbb{P}_{\beta} * \mathbb{P}\left(\dot{T}_{\beta}\right)$.

Note that we assume in the lemma that $\mathbb{P}_{\beta}$ is $\lambda$-distributive, which will only be true under some special circumstances; see the next section. The isomorphism is given by $p \mapsto p \uparrow \beta * p(\beta)$.

Now we consider a factorization of $\mathbb{P}$. Let $\beta<\nu$. Suppose $G_{\beta}$ is a generic filter on $\mathbb{P}_{\beta}$. In $V\left[G_{\beta}\right]$, define a forcing poset $\mathbb{P}_{\beta, \nu}$ as follows. The underlying set of $\mathbb{P}_{\beta, \nu}$ is $\{p \uparrow[\beta, \nu): p \in \mathbb{P}\}$. Let $q \leq p$ in $\mathbb{P}_{\beta, \nu}$ if there exists some $s \in G_{\beta}$ such that $s \cup q \leq s \cup p$ in $\mathbb{P}$. Then $\mathbb{P}$ is isomorphic to a dense subset of $\mathbb{P}_{\beta} * \mathbb{P}_{\beta, \nu}$, by the map which sends $p$ to $p \uparrow \beta * p \uparrow[\beta, \nu)$.

Lemma 5.9. Let $\beta<\nu$. Then $\mathbb{P}_{\beta}$ forces that $\mathbb{P}_{\beta, \nu}$ is $\kappa$-closed.

See Lemma 6.16 of [7] for a proof.

In the next section we will show that under some strong assumptions, any partial square killing forcing iteration on $\lambda$ of length $\nu<\lambda^{+}$is $\lambda$-distributive. Let us observe that this implies that any partial square killing forcing iteration of length $\lambda^{+}$is $\lambda$-distributive.

Lemma 5.10. Let $\mathbb{P}$ be a partial square killing forcing iteration on $\lambda$ of length $\lambda^{+}$, and suppose that for all $\nu<\lambda^{+}, \mathbb{P}_{\nu}$ is $\lambda$-distributive. Then $\mathbb{P}$ is $\lambda$-distributive.

Proof. Let $\dot{f}$ be a nice $\mathbb{P}$-name for a function from $\kappa$ into the ordinals. Then since $\mathbb{P}$ is $\lambda^{+}$-c.c. and $\mathbb{P}=\bigcup\left\{\mathbb{P}_{i}: i<\lambda^{+}\right\}, \dot{f}$ is a $\mathbb{P}_{\nu^{-}}$-name for some $\nu<\lambda^{+}$. By assumption, $\mathbb{P}_{\nu}$ is $\lambda$-distributive, and therefore $\dot{f}$ is forced to be in the ground model.

\section{Distributivity}

We prove that assuming that $\lambda$ is a greatly Mahlo cardinal and $\kappa<\lambda$ is regular and uncountable, then any partial square killing forcing iteration on $\lambda$ of length $\lambda^{+}$ in a generic extension by the Lévy collapse $\operatorname{CoLL}(\kappa,<\lambda)$ is $\lambda$-distributive. The proof is similar to our proof in [7] that a certain forcing iteration in a generic extension by the Lévy collapse is $\lambda$-distributive, assuming that $\lambda$ is weakly compact.

We will use the following two results.

Theorem 6.1 (Magidor [8]). Let $\bar{\lambda}<\lambda$ be strongly inaccessible cardinals, and let $\kappa<\bar{\lambda}$ be regular. Let $G$ be a generic filter on the Lévy collapse $\operatorname{CoLL}(\kappa,<\lambda)$. In $V[G \uparrow \bar{\lambda}]$, let $\mathbb{P}$ be a $\kappa$-closed forcing poset of size less than $\lambda$. Then in $V[G]$, there exists a $V[G \uparrow \bar{\lambda}]$-generic filter $H$ on $\mathbb{P}$ such that

$$
V[G]=V[G \uparrow \bar{\lambda}][H][K],
$$

where $K$ is a $V[G \uparrow \lambda][H]$-generic filter on some $\kappa$-closed forcing poset. 
Note that if $p \in \mathbb{P}$, then the suborder $\mathbb{P} / p=\{q \in \mathbb{P}: q \leq p\}$ satisfies the same assumptions as does $\mathbb{P}$, so we may choose $H$ to contain $p$.

Proposition 6.2. Let $\mu$ be an ordinal of uncountable cofinality, and let $\left\langle d_{\gamma}: \gamma<\mu\right\rangle$ be a sequence such that $d_{\gamma} \subseteq \gamma$ for all $\gamma<\mu$. Let $\mathbb{P}$ be a proper forcing poset. Then $\mathbb{P}$ forces that if $c \subseteq \mu$ is a thread of the sequence $\left\langle d_{\gamma}: \gamma<\mu\right\rangle$, meaning that $c$ is club and for all $\gamma \in \lim (c), c \cap \gamma=d_{\gamma}$, then $c$ is in the ground model.

Proof. Suppose for a contradiction that $p$ forces that $\dot{c}$ is a thread which is not in $V$. Fix some large enough regular cardinal $\theta$, and let $N$ be a countable elementary substructure of $H(\theta)$ which contains as elements $\mathbb{P}, p, \dot{c}$, and $\left\langle d_{\gamma}: \gamma\langle\mu\rangle\right.$. Since $p$ does not decide $\dot{c}$, there is $\xi$ in $N \cap \mu$ and conditions $s, t \leq p$ in $N$ such that $s \Vdash \xi \in \dot{c}$ and $t \Vdash \xi \notin \dot{c}$.

As $\mathbb{P}$ is proper, fix $N$-generic conditions $\bar{s} \leq s$ and $\bar{t} \leq t$. Let $\gamma=\sup (N \cap \mu)$. Then $\gamma<\mu$, since $\mu$ has uncountable cofinality. Now $\bar{s}$ and $\bar{t}$ force that $\sup (N[\dot{G}] \cap$ $\mu)=\sup (N \cap \mu)=\gamma$ is a limit point of $\dot{c}$, and hence that $\dot{c} \cap \gamma=d_{\gamma}$. So $\bar{s}$ and $\bar{t}$ agree about $\dot{c} \cap \gamma$, which contradicts the choice of $\xi$.

Theorem 6.3. Let $\kappa$ be a regular uncountable cardinal, and let $\lambda>\kappa$ be strongly inaccessible. Let $\nu<\lambda^{+}$, and suppose that $\lambda$ is $\nu+1-M a h l o$. Let $G$ be a generic filter on the Lévy collapse $\operatorname{CoLL}(\kappa,<\lambda)$. Then in $V[G]$, any partial square killing forcing iteration on $\lambda$ of length $\nu$ is $\lambda$-distributive.

Proof. We prove the statement by induction on $\lambda$. So let $\lambda>\kappa$ be strongly inaccessible, and assume that the statement of the theorem holds for any strongly inaccessible cardinal $\bar{\lambda}$ with $\kappa<\bar{\lambda}<\lambda$. Let $G$ be a generic filter on $\operatorname{CoLL}(\kappa,<\lambda)$. We prove the following statement by induction on $\nu$ : for all $\nu<\lambda^{+}$, if $\lambda$ is $\nu+1$ Mahlo in $V$, then in $V[G]$ any partial square killing forcing iteration on $\lambda$ of length $\nu$ is $\lambda$-distributive.

Let $\nu<\lambda^{+}$be given, and assume that the statement holds for all $\nu_{0}<\nu$. Suppose that $\lambda$ is $\nu+1$-Mahlo in $V$. In $V[G]$, let $\mathbb{P}$ be a partial square killing forcing iteration on $\lambda$ of length $\nu$, witnessed by a sequence of names $\left\langle\dot{T}_{i}: i<\nu\right\rangle$.

We prove that $\mathbb{P}$ is $\lambda$-distributive. Fix a family $\mathcal{D}$ of dense open subsets of $\mathbb{P}$ with size less than $\lambda$, and fix a condition $p \in \mathbb{P}$. We will find a condition $q \leq p$ in $\cap \mathcal{D}$.

Since $\lambda$ is $\nu+1$-Mahlo in $V$, for all $\beta<\nu, \lambda$ is $\beta+1$-Mahlo in $V$. But $\mathbb{P}_{\beta}$ is a partial square killing forcing iteration on $\lambda$ of length $\beta$, so the induction hypothesis implies that $\mathbb{P}_{\beta}$ is $\lambda$-distributive. Hence all of the proper initial segments of $\mathbb{P}$ are $\lambda$-distributive.

Fix a sequence of names

$$
\left\langle\dot{c}_{\alpha}^{\beta}: \beta<\nu, \alpha<\lambda\right\rangle
$$

in $V[G]$ such that for all $\beta<\nu$ and $\alpha<\lambda$,

$$
\mathbb{P}_{\beta} \Vdash \dot{c}_{\alpha}^{\beta} \neq \emptyset \Longleftrightarrow \alpha \in \dot{T}_{\beta},
$$

and

$$
\mathbb{P}_{\beta} \Vdash\left\langle\dot{c}_{\alpha}^{\beta}: \alpha \in \dot{T}_{\beta}\right\rangle \text { is a partial square sequence. }
$$

Fix a $\operatorname{CoLL}(\kappa,<\lambda)$-name $\dot{x}$ in $H\left(\lambda^{+}\right)^{V}$ such that

$$
\dot{x}^{G}=\left\langle\mathbb{P}, p, \mathcal{D},\left\langle\dot{T}_{\beta}: \beta<\nu\right\rangle,\left\langle\dot{c}_{\alpha}^{\beta}: \beta<\nu, \alpha<\lambda\right\rangle\right\rangle .
$$

This is possible, as $\nu<\lambda^{+}$and $H\left(\lambda^{+}\right)^{V[G]}=H\left(\lambda^{+}\right)^{V}[G]$.

Recall that in $V, S_{\nu}^{\lambda}$ is the collection of all $N$ satisfying: 
(1) $N \prec H\left(\lambda^{+}\right)$;

(2) $N \cap \lambda=\lambda_{N}$ is strongly inaccessible;

(3) $|N|=\lambda_{N}$;

(4) $N^{<\lambda_{N}} \subseteq N$;

(5) there is a canonical function $f_{\nu}$ on $\lambda$ of $\operatorname{rank} \nu$ in $N$ such that $\lambda_{N}$ is $f_{\nu}\left(\lambda_{N}\right)$ Mahlo.

Since $\lambda$ is $\nu+1$-Mahlo in $V$, by Lemma 3.3 the set $S_{\nu}^{\lambda}$ is a stationary subset of $P_{\lambda}\left(H\left(\lambda^{+}\right)\right)$in $V$. Fix $N$ in $S_{\nu}^{\lambda}$ such that

$$
N \prec\left(H\left(\lambda^{+}\right)^{V}, \in, \kappa, \nu, \dot{x}\right) \text {. }
$$

Fix a canonical function $f_{\nu}$ on $\lambda$ of rank $\nu$ in $N$ such that $\lambda_{N}$ is $f_{\nu}\left(\lambda_{N}\right)$-Mahlo.

Let

$$
\pi: N \rightarrow \bar{N}
$$

be the transitive collapsing map of $N$ in $V$. By Lemma 3.6,

$$
\pi(\nu)=f_{\nu}\left(\lambda_{N}\right)
$$

Let

$$
\sigma: N[G] \rightarrow \overline{N[G]}
$$

be the transitive collapsing map of $N[G]$ in $V[G]$. By Lemma 3.8,

$$
\sigma \uparrow N=\pi
$$

and

$$
\overline{N[G]}=\bar{N}\left[G \uparrow \lambda_{N}\right] .
$$

In particular, $\overline{N[G]}$ is in $V\left[G\left\lceil\lambda_{N}\right]\right.$, and is a $\lambda_{N}$-model. Note that

$$
\sigma(\lambda)=\lambda_{N} \text {. }
$$

Since $\dot{x}$ is in $N$, the sets

$$
\mathbb{P}, p, \mathcal{D},\left\langle\dot{T}_{\beta}: \beta<\nu\right\rangle,\left\langle\dot{c}_{\alpha}^{\beta}: \beta<\nu, \alpha<\lambda\right\rangle
$$

are in $N[G]$. As $\mathcal{D}$ has size less than $\lambda, \mathcal{D} \subseteq N[G]$.

Let $\theta(a, b, c)$ be the formula of LST described in Section 4 which asserts that $a$ is a partial square killing forcing iteration on $b$ of length $c$. Since $N[G] \prec H\left(\lambda^{+}\right)^{V[G]}$,

$$
N[G] \models \theta(\mathbb{P}, \lambda, \nu) .
$$

As $\sigma$ is an isomorphism,

$$
\bar{N}\left[G \nmid \lambda_{N}\right] \models \theta\left(\sigma(\mathbb{P}), \lambda_{N}, f_{\nu}\left(\lambda_{N}\right)\right) .
$$

Since $\bar{N}\left[G \uparrow \lambda_{N}\right]$ is a $\lambda_{N}$-model, it follows from Lemma 4.6 that $\sigma(\mathbb{P})$ is a partial square killing forcing iteration on $\lambda_{N}$ of length $f_{\nu}\left(\lambda_{N}\right)$ in $V\left[G\left\lceil\lambda_{N}\right]\right.$.

Now $\lambda_{N}$ is $f_{\nu}\left(\lambda_{N}\right)$-Mahlo in $V$. So for all $\zeta<f_{\nu}\left(\lambda_{N}\right), \lambda_{N}$ is $\zeta+1$-Mahlo in $V$. By the induction hypothesis applied to $\lambda_{N}$,

$$
\forall \zeta<f_{\nu}\left(\lambda_{N}\right)\left(\sigma(\mathbb{P})_{\zeta} \text { is } \lambda_{N} \text {-distributive in } V\left[G \uparrow \lambda_{N}\right]\right) \text {. }
$$

Since $\sigma(\mathbb{P})$ is $\kappa$-closed, by Magidor's Theorem there exists a $V\left[G\left\lceil\lambda_{N}\right]\right.$-generic filter $\bar{H}$ on $\sigma(\mathbb{P})$ containing $\sigma(p)$ such that

$$
V[G]=V\left[G \uparrow \lambda_{N}\right][\bar{H}][K],
$$

where $K$ is a $V\left[G \nmid \lambda_{N}\right][\bar{H}]$-generic filter on some $\kappa$-closed forcing poset in $V[G \uparrow$ $\left.\lambda_{N}\right][\bar{H}]$. 
Note that

$$
\sigma \uparrow(\mathbb{P} \cap N[G])
$$

is an isomorphism between the partial orderings $\mathbb{P} \cap N[G]$ and $\sigma(\mathbb{P})$. Define

$$
H=\sigma^{-1}(\bar{H}) \text {. }
$$

Then $H$ is a filter on $\mathbb{P} \cap N[G]$, and $H$ is $N[G]$-generic for $\mathbb{P}$. For if $D$ is a dense subset of $\mathbb{P}$ in $N[G]$, then $\sigma(D)$ is a dense subset of $\sigma(\mathbb{P})$ in $V\left[G \uparrow \lambda_{N}\right]$. As $\bar{H}$ is $V\left[G \uparrow \lambda_{N}\right]$-generic for $\sigma(\mathbb{P})$, fix $s$ in $\bar{H} \cap \sigma(D)$. Then $\sigma^{-1}(s)$ is in $D \cap H \cap N[G]$.

Note that if $u \in H$, then $\operatorname{dom}(u) \subseteq N[G]$, since $\operatorname{dom}(u)$ has size less than $\lambda$. It follows easily from this fact and the $N[G]$-genericity of $H$ that

$$
\bigcup\{\operatorname{dom}(u): u \in H\}=N[G] \cap \nu=N \cap \nu .
$$

Similarly, if $u \in H$ and $\beta \in \operatorname{dom}(u)$, then $u(\beta)$ is a subset of $N[G] \cap \lambda=\lambda_{N}$.

Define a partial function $q: \nu \rightarrow X$ with domain $N[G] \cap \nu$ as follows. Given $\beta$ in $N[G] \cap \nu$, by the compatibility of conditions in $H$ and the $N[G]$-genericity of $H$, the set

$$
\bigcup\{u(\beta): u \in H\}
$$

is a cofinal subset $\lambda_{N}$ which is closed below $\lambda_{N}$. Define

$$
q(\beta)=\bigcup\{u(\beta): u \in H\} \cup\left\{\lambda_{N}\right\} \text {. }
$$

Then $q(\beta)$ is in $X_{\lambda}$. Since $|\operatorname{dom}(q)|=|N \cap \nu| \leq|N|<\lambda, q$ is in $X_{\lambda, \nu}$.

We will be done if we can show that $q$ is in $\mathbb{P}$. For then by the definition of $q$, $q \leq u$ for all $u$ in $H$. Also $p \in H$, since $\sigma(p) \in \bar{H}$, and therefore $q \leq p$. To show $q \in \bigcap \mathcal{D}$, let $D \in \mathcal{D}$ be given. Then $D$ is in $N[G]$, so by the $N[G]$-genericity of $H$, fix $u$ in $D \cap H$. Then $q \leq u$, so $q$ is in $D$, since $D$ is open. This completes the proof.

Suppose for a contradiction that $q$ is not a condition in $\mathbb{P}$. Since $q$ is in $X_{\lambda, \nu}$, by Lemma 5.4 it follows that there is some $\beta$ in $\operatorname{dom}(q)$ such that $q \uparrow \beta$ is in $\mathbb{P}_{\beta}$, but $q \uparrow \beta$ does not force that $q(\beta)$ is disjoint from $\dot{T}_{\beta}$.

Define

$$
H_{\beta}=\{u \uparrow \beta: u \in H\} .
$$

Since $H$ is upwards closed in $\mathbb{P} \cap N[G], H_{\beta} \subseteq H$, and therefore

$$
H_{\beta}=H \cap \mathbb{P}_{\beta} \text {. }
$$

Also it follows from the $N[G]$-genericity of $H$ and an easy argument using Lemma 5.3(2) that $H_{\beta}$ is $N[G]$-generic for $\mathbb{P}_{\beta}$.

Note that $q \uparrow \beta \leq u$ in $\mathbb{P}_{\beta}$ for all $u$ in $H_{\beta}$. It follows that $q \uparrow \beta$ forces that every proper initial segment of $q(\beta)$ is disjoint from $\dot{T}_{\beta}$. Since $q \uparrow \beta$ does not force that $q(\beta)$ is disjoint from $\dot{T}_{\beta}$, there is a condition below $q \uparrow \beta$ in $\mathbb{P}_{\beta}$ which forces that $\lambda_{N}$ is in $\dot{T}_{\beta}$.

Let

It is not hard to show that

$$
\bar{H}_{\sigma(\beta)}=\sigma\left[H_{\beta}\right]
$$

$$
\bar{H}_{\sigma(\beta)}=\{s \uparrow \sigma(\beta): s \in \bar{H}\}=\bar{H} \cap \sigma(\mathbb{P})_{\sigma(\beta)} .
$$

It follows by Lemma $5.3(3)$ that $\bar{H}_{\sigma(\beta)}$ is a $V\left[G \uparrow \lambda_{N}\right]$-generic filter on $\sigma(\mathbb{P})_{\sigma(\beta)}$. Let

$$
\bar{H}_{\sigma(\beta), \sigma(\nu)}=\{s \uparrow[\sigma(\beta), \sigma(\nu)): s \in \bar{H}\} .
$$


Then $\bar{H}_{\sigma(\beta), \sigma(\nu)}$ is a $V\left[G \uparrow \lambda_{N}\right]\left[\bar{H}_{\sigma(\beta)}\right]$-generic filter on $\sigma(\mathbb{P})_{\sigma(\beta), \sigma(\nu)}$, and

$$
V[G]=V\left[G \uparrow \lambda_{N}\right]\left[\bar{H}_{\sigma(\beta)}\right]\left[\bar{H}_{\sigma(\beta), \sigma(\nu)}\right][K] .
$$

By Lemma 5.9, it follows that $V[G]$ is a generic extension of $V\left[G \uparrow \lambda_{N}\right]\left[\bar{H}_{\sigma(\beta)}\right]$ by a $\kappa$-closed forcing poset.

Recall that

$$
\mathbb{P}_{\beta} \Vdash\left\langle\dot{c}_{\alpha}^{\beta}: \alpha \in \dot{T}_{\beta}\right\rangle
$$

is a partial square sequence. Fix a sequence of $\mathbb{P}_{\beta}$-names

$$
\left\langle\dot{d}_{\gamma}: \gamma<\lambda\right\rangle
$$

in $N[G]$ satisfying that for all $\gamma<\lambda$,

$$
\mathbb{P}_{\beta} \Vdash \dot{d}_{\gamma}= \begin{cases}\dot{c}_{\alpha}^{\beta} \cap \gamma & \text { if } \gamma \in \lim \left(\dot{c}_{\alpha}^{\beta}\right) \cap \alpha \\ \emptyset & \text { otherwise. }\end{cases}
$$

Note that by coherence, the definition of $\dot{d}_{\gamma}$ in the first case is independent of $\alpha$.

For each $\gamma<\lambda, \mathbb{P}_{\beta}$ forces that $\dot{d}_{\gamma}$ has order type less than $\kappa$. But $\mathbb{P}_{\beta}$ is $\kappa$-closed, so $\mathbb{P}_{\beta}$ forces that $\dot{d}_{\gamma}$ is in the ground model $V[G]$. By the $N[G]$-genericity of $H_{\beta}$, for each $\gamma<\lambda_{N}$ we can fix $d_{\gamma}$ in $N[G]$ such that

$$
\exists u \in H_{\beta}\left(u \Vdash_{\mathbb{P}_{\beta}} \dot{d}_{\gamma}=\check{d}_{\gamma}\right) .
$$

This defines a sequence

$$
\left\langle d_{\gamma}: \gamma<\lambda_{N}\right\rangle
$$

in the model $V[G]$.

Recall that there is a condition below $q \uparrow \beta$ in $\mathbb{P}_{\beta}$ which forces that $\lambda_{N}$ is in $\dot{T}_{\beta}$, and hence that $\dot{c}_{\lambda_{N}}^{\beta}$ is a club subset of $\lambda_{N}$ of order type $\kappa$. By the induction hypothesis, $\mathbb{P}_{\beta}$ is $\lambda$-distributive. So we can find $t \leq q \uparrow \beta$ in $\mathbb{P}_{\beta}$ and a club set $c \subseteq \lambda_{N}$ with order type $\kappa$ such that

$$
t \Vdash_{\mathbb{P}_{\beta}} \dot{c}_{\lambda_{N}}^{\beta}=\check{c} .
$$

Then

$$
\forall \gamma \in \lim (c) \cap \lambda_{N}\left(t \Vdash_{\mathbb{P}_{\beta}} \check{c} \cap \gamma=\dot{d}_{\gamma}\right) .
$$

But $t$ is a lower bound of $H \uparrow \beta$. So

$$
\forall \gamma \in \lim (c) \cap \lambda_{N}\left(t \Vdash_{\mathbb{P}_{\beta}} \check{c} \cap \gamma=\check{d}_{\gamma}\right) .
$$

Therefore

$$
\forall \gamma \in \lim (c) \cap \lambda_{N}\left(c \cap \gamma=d_{\gamma}\right)
$$

In other words, $c$ is a thread of the sequence $\left\langle d_{\gamma}: \gamma\left\langle\lambda_{N}\right\rangle\right.$.

We claim that the sequence $\left\langle d_{\gamma}: \gamma<\lambda_{N}\right\rangle$ is in the model $V\left[G \uparrow \lambda_{N}\right]\left[\bar{H}_{\sigma(\beta)}\right]$. The sequence of names $\left\langle\dot{d}_{\gamma}: \gamma<\lambda\right\rangle$ is in $N[G]$, and clearly

$$
\sigma\left(\left\langle\dot{d}_{\gamma}: \gamma<\lambda\right\rangle\right)=\left\langle\sigma\left(\dot{d}_{\gamma}\right): \gamma<\lambda_{N}\right\rangle .
$$

For all $\gamma<\lambda_{N}$, there is some $u$ in $H_{\beta}$ such that

$$
u \Vdash_{\mathbb{P}_{\beta}} \dot{d}_{\gamma}=\check{d}_{\gamma} .
$$

Then

It follows that

$$
N[G] \models u \Vdash_{\mathbb{P}_{\beta}} \dot{d}_{\gamma}=\check{d}_{\gamma} .
$$

$$
\bar{N}\left[G \uparrow \lambda_{N}\right] \models \sigma(u) \Vdash_{\sigma\left(\mathbb{P}_{\beta}\right)} \sigma\left(\dot{d}_{\gamma}\right)=\sigma\left(\check{d}_{\gamma}\right) .
$$


Therefore in $V\left[G \uparrow \lambda_{N}\right]$,

$$
\sigma(u) \Vdash_{\sigma\left(\mathbb{P}_{\beta}\right)} \sigma\left(\dot{d}_{\gamma}\right)=\sigma\left(\check{d}_{\gamma}\right) .
$$

Also note that $\sigma(u) \in \bar{H}_{\sigma(\beta)}$. Since $d_{\gamma}$ is a bounded subset of $\lambda_{N}, \sigma\left(d_{\gamma}\right)=d_{\gamma}$. It follows that the sequence $\left\langle d_{\gamma}: \gamma<\lambda_{N}\right\rangle$ is the $\bar{H}_{\sigma(\beta)}$-interpretation of $\left\langle\sigma\left(\dot{d}_{\gamma}\right): \gamma<\right.$ $\left.\lambda_{N}\right\rangle$, considered as a $\sigma\left(\mathbb{P}_{\beta}\right)$-name. Thus this sequence is in $V\left[G \uparrow \lambda_{N}\right]\left[\bar{H}_{\sigma(\beta)}\right]$, as claimed.

In the model $V\left[G\left\lceil\lambda_{N}\right], \lambda_{N}\right.$ is equal to $\kappa^{+}$. For all $\zeta<f_{\nu}\left(\lambda_{N}\right), \sigma(\mathbb{P})_{\zeta}$ is $\lambda_{N}{ }^{-}$ distributive by the induction hypothesis. But $\beta<\nu$, so $\sigma(\beta)<\sigma(\nu)=f_{\nu}\left(\lambda_{N}\right)$. It follows that $\sigma\left(\mathbb{P}_{\beta}\right)=\sigma(\mathbb{P})_{\sigma(\beta)}$ is $\lambda_{N}$-distributive in $V\left[G\left\lceil\lambda_{N}\right]\right.$. So $\lambda_{N}$ is equal to $\kappa^{+}$in the model $V\left[G \uparrow \lambda_{N}\right]\left[\bar{H}_{\sigma(\beta)}\right]$.

In the model $V\left[G \uparrow \lambda_{N}\right]\left[\bar{H}_{\sigma(\beta)}\right]$, we have a sequence $\left\langle d_{\gamma}: \gamma<\lambda_{N}\right\rangle$, where each $d_{\gamma}$ is a subset of $\gamma$. In the model $V[G], c$ is a thread of this sequence. But $V[G]$ is a generic extension of $V\left[G \nmid \lambda_{N}\right]\left[\bar{H}_{\sigma(\beta)}\right]$ by a $\kappa$-closed forcing poset. By Proposition 6.2 , it follows that $c$ is in $V\left[G\left\lceil\lambda_{N}\right]\left[\bar{H}_{\sigma(\beta)}\right]\right.$. But $c$ has order type $\kappa$ and is cofinal in $\lambda_{N}$. This contradicts that $\lambda_{N}$ is equal to $\kappa^{+}$in the model $V\left[G\left\lceil\lambda_{N}\right]\left[\bar{H}_{\sigma(\beta)}\right]\right.$. This contradiction shows that $q$ must be a condition in $\mathbb{P}$ as desired, and the proof is complete.

\section{No Partial Squares from a Greatly Mahlo Cardinal}

We are now ready to construct a model with no partial square sequences. Let $V$ be a model of set theory satisfying the following assumptions:

(1) $\kappa$ is a regular uncountable cardinal;

(2) $\lambda$ is a greatly Mahlo cardinal larger than $\kappa$;

(3) $2^{\lambda}=\lambda^{+}$.

We begin by Lévy collapsing $\lambda$ to become $\kappa^{+}$. So let $G$ be a generic filter on the Lévy collapse $\operatorname{CoLL}(\kappa,<\lambda)$. Then in $V[G], 2^{\kappa}=\lambda=\kappa^{+}$and $2^{\lambda}=\lambda^{+}$.

Working in $V[G]$, we define by recursion sequences

$$
\left\langle\mathbb{P}_{i}: i \leq \lambda^{+}\right\rangle
$$

and

$$
\left\langle\dot{T}_{j}^{i}: i, j<\lambda^{+}\right\rangle .
$$

For bookkeeping purposes, fix a function $f: \lambda^{+} \rightarrow \lambda^{+} \times \lambda^{+}$satisfying that $f(\alpha)=$ $\langle i, j\rangle$ implies $i \leq \alpha$. We also define a sequence

$$
\left\langle\dot{T}_{\alpha}: \alpha<\lambda^{+}\right\rangle
$$

by letting $\dot{T}_{\alpha}=\dot{T}_{j}^{i}$, where $f(\alpha)=\langle i, j\rangle$.

We will maintain the following recursion hypotheses: for all $\beta \leq \lambda^{+}$,

(1) $\mathbb{P}_{\beta}$ is a partial square killing forcing iteration on $\lambda$ of length $\beta$, witnessed by the sequence of names $\left\langle\dot{T}_{\alpha}: \alpha<\beta\right\rangle$;

(2) for all $\zeta<\beta, \mathbb{P}_{\zeta}=\mathbb{P}_{\beta}\lceil\zeta$;

(3) if $\beta<\lambda^{+}$, then $\left\langle\dot{T}_{i}^{\beta}: i<\lambda^{+}\right\rangle$is a list of all nice $\mathbb{P}_{\beta}$-names for subsets of $\lambda \cap \operatorname{cof}(\kappa)$ which are forced by $\mathbb{P}_{\beta}$ to carry a partial square.

Let us summarize the basic properties which $\mathbb{P}_{\beta}$ will satisfy if the above hypotheses hold:
(a) $\mathbb{P}_{\beta}$ is $\kappa$-closed (by Lemma 5.6 );
(b) $\left|\mathbb{P}_{\beta}\right|<\lambda^{+}$if $\beta<\lambda^{+}$(by Lemma 5.7); 
(c) $\mathbb{P}_{\beta}$ is $\lambda^{+}$-c.c. (by Lemma 5.7);

(d) $\mathbb{P}_{\beta}$ is $\lambda$-distributive (by Theorem 6.3 and Lemma 5.10);

(e) if $\beta=\gamma+1$, then $\mathbb{P}_{\beta}$ is forcing equivalent to $\mathbb{P}_{\gamma} * \mathbb{P}\left(\dot{T}_{\gamma}\right)$ (by Lemma 5.8).

Let $\mathbb{P}_{0}$ be the trivial forcing consisting of just the empty function.

Suppose $\nu<\lambda^{+}$and $\mathbb{P}_{\beta}$ is defined for all $\beta \leq \nu$. Also assume $\left\langle\dot{T}_{i}^{\beta}: i<\lambda^{+}\right\rangle$is defined for all $\beta<\nu$. Choose a sequence $\left\langle\dot{T}_{i}^{\nu}: i<\lambda^{+}\right\rangle$satisfying (3) above. This is possible, as $2^{\lambda}=\lambda^{+}$and (b) imply that there are only $\lambda^{+}$many nice $\mathbb{P}_{\nu}$-names for a subset of $\lambda$.

Let $f(\nu)=\langle i, j\rangle$. Then $i \leq \nu$, so $\dot{T}_{j}^{i}$ is defined. Let $\dot{T}_{\nu}=\dot{T}_{j}^{i}$. Then $\dot{T}_{\nu}$ is a nice $\mathbb{P}_{i}$-name for a subset of $\lambda \cap \operatorname{cof}(\kappa)$ which is forced to carry a partial square. The property of carrying a partial square is easily seen to be upwards absolute. So $\dot{T}_{\nu}$ is a nice $\mathbb{P}_{\nu}$-name for a subset of $\lambda \cap \operatorname{cof}(\kappa)$ which carries a partial square.

Now define $\mathbb{P}_{\nu+1}$ as follows. The underlying set of $\mathbb{P}_{\nu+1}$ consists of all functions $p$ in $X_{\lambda, \nu+1}$ satisfying that $p\left\lceil\nu \in \mathbb{P}_{\nu}\right.$, and if $\nu \in \operatorname{dom}(p)$, then $p\left\lceil\nu \Vdash_{\mathbb{P}_{\nu}} p(\nu) \cap \dot{T}_{\nu}=\emptyset\right.$. The ordering on $\mathbb{P}_{\nu+1}$ is by $\leq_{\lambda, \nu+1}$.

Let $\nu \leq \lambda^{+}$be a limit ordinal, and suppose that $\mathbb{P}_{\beta}$ is defined for all $\beta<\nu$. Define $\mathbb{P}_{\nu}$ as the suborder of $X_{\lambda, \nu}$ consisting of functions $p \in X_{\lambda, \nu}$ such that $p\left\lceil\beta \in \mathbb{P}_{\beta}\right.$ for all $\beta<\nu$.

Now we verify the recursion hypotheses. (3) is clear. Let us prove (2). This is trivial for $\mathbb{P}_{0}$. Consider $\mathbb{P}_{\nu+1}$. Clearly $\mathbb{P}_{\nu} \subseteq \mathbb{P}_{\nu+1}$, and therefore $\mathbb{P}_{\nu+1}\left\lceil\nu=\mathbb{P}_{\nu}\right.$. Then for all $\beta<\nu, \mathbb{P}_{\nu+1}\left\lceil\beta=\mathbb{P}_{\nu}\left\lceil\beta=\mathbb{P}_{\beta}\right.\right.$, by the recursion hypotheses. Similarly for a limit ordinal $\nu$ and $\beta<\nu, \mathbb{P}_{\beta} \subseteq \mathbb{P}_{\nu}$ follows easily from the definition of $\mathbb{P}_{\nu}$ and the recursion hypotheses. This implies $\mathbb{P}_{\nu} \uparrow \beta=\mathbb{P}_{\beta}$.

It remains to prove (1), by verifying the properties of Definition 4.5. (1)-(4) are immediate from the definition and the recursion hypotheses. The verification of (5) is tedious, but completely trivial. So we will omit it.

This completes the construction. Let $\mathbb{P}=\mathbb{P}_{\lambda^{+}}$.

Let $H$ be a $V[G]$-generic filter on $\mathbb{P}$. Then in $V[G][H], \lambda=\kappa^{+}$. Suppose $T \subseteq \lambda \cap \operatorname{cof}(\kappa)$ carries a partial square in $V[G][H]$. Then in $V[G]$, there is a nice $\mathbb{P}$-name $\dot{T}$ for a subset of $\lambda \cap \operatorname{cof}(\kappa)$ such that $\dot{T}^{G}=T$ and $\mathbb{P}$ forces that $\dot{T}$ carries a partial square.

Since $\mathbb{P}$ is $\lambda^{+}$-c.c. and $\mathbb{P}=\bigcup\left\{\mathbb{P}_{i}: i<\lambda^{+}\right\}$, we can find $\alpha<\lambda^{+}$such that $\dot{T}$ is a nice $\mathbb{P}_{\alpha}$-name for a subset of $\lambda \cap \operatorname{cof}(\kappa)$, and moreover $\mathbb{P}_{\alpha}$ forces that $\dot{T}$ carries a partial square. So for some $i<\lambda^{+}, \dot{T}=\dot{T}_{i}^{\alpha}$. Now choose $\nu \geq \alpha$ such that $f(\nu)=\langle\alpha, i\rangle$. Then $\dot{T}=\dot{T}_{i}^{\alpha}=\dot{T}_{\nu}$. But $\mathbb{P}_{\nu+1}$ is forcing equivalent to $\mathbb{P}_{\nu} * \mathbb{P}\left(\dot{T}_{\nu}\right)$. So in $V\left[G \cap \mathbb{P}_{\nu+1}\right], T$ is non-stationary. Thus $T$ is non-stationary in $V[G]$.

We have shown that in $V[G]$, no stationary subset of $\kappa^{+} \cap \operatorname{cof}(\kappa)$ carries a partial square, which completes the proof.

\section{A Related Equiconsistency Result}

Let $\lambda$ be a regular uncountable cardinal. Recall the principle $\square(\lambda)$, which asserts the existence of a sequence

$$
\left\langle c_{\alpha}: \alpha<\lambda, \alpha \text { limit }\right\rangle
$$


satisfying:

(1) $c_{\alpha}$ is a club subset of $\alpha$;

(2) if $\gamma$ is a limit point of $c_{\alpha}$, then $c_{\alpha} \cap \gamma=c_{\gamma}$;

(3) there does not exist a club set $C \subseteq \lambda$ such that for all $\gamma$ in $\lim (C), C \cap \gamma=c_{\gamma}$.

As with partial squares, we can relativize this idea to a stationary set.

Definition 8.1. Let $\lambda$ be a regular uncountable cardinal, and let $A \subseteq \lambda$. The principle $\square(\lambda, A)$ asserts the existence of a sequence

$$
\left\langle c_{\alpha}: \alpha \in A\right\rangle
$$

satisfying:

(1) $c_{\alpha}$ is a club subset of $\alpha$;

(2) if $\gamma$ is a limit point of $c_{\alpha}$ and $c_{\beta}$, then $c_{\alpha} \cap \gamma=c_{\beta} \cap \gamma$;

(3) there does not exist a club set $C \subseteq \lambda$ satisfying that whenever $\gamma$ is in $\lim (C)$, there exists $\alpha \in A$ such that $\gamma \in \lim \left(c_{\alpha}\right)$ and $C \cap \gamma=c_{\alpha} \cap \gamma$.

Suppose $\left\langle c_{\alpha}: \alpha \in A\right\rangle$ is a sequence satisfying (1) and (2). Define $\left\langle d_{\gamma}: \gamma<\lambda\right\rangle$ by

$$
d_{\gamma}= \begin{cases}c_{\alpha} \cap \gamma & \text { if } \gamma \in \lim \left(c_{\alpha}\right) \\ \emptyset & \text { otherwise. }\end{cases}
$$

Let us call $\left\langle d_{\gamma}: \gamma\langle\lambda\rangle\right.$ the derived sequence of $\left\langle c_{\alpha}: \alpha \in A\right\rangle$. Then property (3) is equivalent to the assertion that the sequence $\left\langle d_{\gamma}: \gamma\langle\lambda\rangle\right.$ does not have a thread, that is, there does not exist a club $C \subseteq \lambda$ such that for all $\gamma$ in $\lim (C), C \cap \gamma=d_{\gamma}$.

Theorem 8.2. The statement that there exists a regular uncountable cardinal $\kappa$ such that $\neg \square\left(\kappa^{+}, A\right)$ holds for every stationary set $A \subseteq \kappa^{+} \cap \operatorname{cof}(\kappa)$ is equiconsistent with a weakly compact cardinal.

Jensen showed that the failure of $\square\left(\kappa^{+}\right)$implies that $\kappa^{+}$is weakly compact in $L$ ([11]), and this proves the lower bound. Veličković [15] showed that if $\lambda$ is weakly compact and $\kappa<\lambda$ is regular and uncountable, then $\operatorname{CoLL}(\kappa,<\lambda)$ forces $\neg \square\left(\kappa^{+}\right)$ (also see [11]). A variation of the forcing construction given above will show how to obtain $\neg \square\left(\kappa^{+}, A\right)$ for any stationary set $A \subseteq \kappa^{+} \cap \operatorname{cof}(\kappa)$.

We will use a characterization of weakly compact cardinals which was proven by the first author in [7].

Theorem 8.3. Let $\lambda$ be a regular uncountable cardinal. Then $\lambda$ is weakly compact iff there are stationarily many $N$ in $P_{\lambda}\left(H\left(\lambda^{+}\right)\right)$satisfying:

(1) $N \prec H\left(\lambda^{+}\right)$;

(2) $N \cap \lambda=\lambda_{N}$ is strongly inaccessible;

(3) $N^{<\lambda_{N}} \subseteq N$;

(4) $\bar{N} \prec_{1} H\left(\lambda_{N}^{+}\right)$.

The forcing construction we use to prove Theorem 8.2 is nearly identical to that given in the previous sections, so we will only point out the differences.

The ground model $V$ satisfies that $\lambda$ is weakly compact. We define a forcing iteration in $V[G]$, where $G$ is a generic filter on $\operatorname{CoLL}(\kappa,<\lambda)$. Given $\mathbb{P}_{\beta}$, enumerate all nice $\mathbb{P}_{\beta}$-names for a subset of $\lambda \cap \operatorname{cof}(\kappa)$ as $\left\langle\dot{T}_{\alpha}^{\beta}: \alpha<\lambda^{+}\right\rangle$. Let $f(\beta)=\langle i, j\rangle$, where $f$ is a bookkeeping function. If $\mathbb{P}_{\beta}$ forces $\square\left(\kappa^{+}, \dot{T}_{j}^{i}\right)$, then let $\dot{T}_{\beta}=\dot{T}_{j}^{i}$. Otherwise let $\dot{T}_{\beta}$ be a name for the empty set. The rest of the definition of the forcing iteration is the same as before. One now shows easily that the forcing 
iteration satisfies Definition 4.5(1,2,3,5), and thus all the properties discussed in Section 5 .

The proof that the iteration is $\lambda$-distributive is by induction. So assume $\mathbb{P}_{\beta}$ is $\lambda$-distributive for all $\beta<\nu$, and we show that $\mathbb{P}_{\nu}$ is $\lambda$-distributive. Again we choose an elementary substructure $N$, but this time we assume that it satisfies the properties listed in Definition 8.3. The definition of $q$ is as before, and it will suffice to show $q$ is a condition. Suppose for a contradiction it is not, and let $\beta$ in $\operatorname{dom}(q)$ be a counterexample.

Fix a name $\left\langle\dot{c}_{\beta}: \beta \in \dot{T}_{\beta}\right\rangle$ in $N$ which $\mathbb{P}_{\beta}$ forces satisfies Definition 8.1(1,2,3). Let $\left\langle\dot{d}_{\gamma}: \gamma<\lambda\right\rangle$ be a name for the derived sequence of $\left\langle\dot{c}_{\beta}: \beta \in \dot{T}_{\beta}\right\rangle$. Then $\mathbb{P}_{\beta}$ forces that the sequence $\left\langle\dot{d}_{\gamma}: \gamma<\lambda\right\rangle$ does not have a thread. This last statement can be shown to be $\Pi_{1}$. Since $\sigma$ is an isomorphism and $\bar{N}\left[G \nmid \lambda_{N}\right] \prec_{1} H\left(\lambda_{N}^{+}\right)^{V\left[G\left\lceil\lambda_{N}\right]\right.}$, in the model $V\left[G\left\lceil\lambda_{N}\right], \sigma\left(\mathbb{P}_{\beta}\right)\right.$ forces that $\sigma\left(\left\langle\dot{d}_{\gamma}: \gamma<\lambda\right\rangle\right)$ does not have a thread.

By the induction hypothesis, $\mathbb{P}_{\beta}$ is $\lambda$-distributive. But the property of being $\lambda$-distributive is $\Pi_{1}$. So again the fact that $\bar{N}\left[G\left\lceil\lambda_{N}\right] \prec_{1} H\left(\lambda_{N}^{+}\right)^{V\left[G\left\lceil\lambda_{N}\right]\right.}\right.$ implies that $\sigma\left(\mathbb{P}_{\beta}\right)$ is $\lambda_{N}$-distributive in $V\left[G\left\lceil\lambda_{N}\right]\right.$. Therefore $\lambda_{N}$ is equal to $\kappa^{+}$in $V[G \uparrow$ $\left.\lambda_{N}\right]\left[\bar{H}_{\sigma(\beta)}\right]$.

As in the proof of Theorem 6.3, we define a sequence $\left\langle d_{\gamma}: \gamma<\lambda_{N}\right\rangle$ which turns out to be the $\bar{H}_{\sigma(\beta)}$-interpretation of $\sigma\left(\left\langle\dot{d}_{\gamma}: \gamma<\lambda\right\rangle\right)$, and find a club $c \subseteq \lambda_{N}$ in $V[G]$ which threads this sequence. An application of Proposition 6.2 shows that the thread $c$ is in $V\left[G\left\lceil\lambda_{N}\right]\left[\bar{H}_{\sigma(\beta)}\right]\right.$. This contradicts that $\sigma\left(\mathbb{P}_{\beta}\right)$ forces in $V\left[G\left\lceil\lambda_{N}\right]\right.$ that $\sigma\left(\left\langle\dot{d}_{\gamma}: \gamma<\lambda\right\rangle\right)$ does not have a thread.

Remark. The results of this paper are related in some ways to Mitchell's construction of a model in which there is no stationary subset of $\kappa^{+} \cap \operatorname{cof}(\kappa)$ in the approachability ideal $I\left[\kappa^{+}\right]$, using a greatly Mahlo cardinal ([10]). In this model (and also in the model constructed in [9]), there is no stationary subset of $\kappa^{+} \cap \operatorname{cof}(\kappa)$ which carries a partial square. However, Mitchell's argument works only when $\kappa=\mu^{+}$ for some regular cardinal $\mu$. Our forcing construction, on the other hand, assumes only that $\kappa$ is regular and uncountable. Another difference is that GCH holds in our model, whereas in Mitchell's model, $2^{\mu}=\mu^{++}$.

\section{REFERENCES}

[1] J. Baumgartner. Iterated forcing. In Surveys in Set Theory, pages 1-59. Cambridge Univ. Press, 1983.

[2] J. Baumgartner, A. Taylor, and S. Wagon. On splitting stationary subsets of large cardinals. J. Symbolic Logic, 42(2):203-214, 1977.

[3] D. Burke. Generic embeddings and the failure of box. Proc. Amer. Math. Soc., 123(9):28672871,1995

[4] T. Jech. Stationary subsets of inaccessible cardinals. In Axiomatic set theory, volume 31 of Contemp. Math., pages 115-142. Amer. Math. Soc., Providence, RI, 1984.

[5] T. Jech. Set theory. The third millennium edition, revised and expanded. Springer Monographs in Mathematics. Springer-Verlag, Berlin, 2003.

[6] R. Jensen. The fine structure of the constructible hierarchy. Ann. Math. Logic, 4:229-308, 1972.

[7] J. Krueger. Weak compactness and no partial squares. To appear in J. Symbolic Logic.

[8] M. Magidor. Reflecting stationary sets. J. Symbolic Logic, 47(4):755-771, 1982.

[9] W. Mitchell. A weak variation of Shelah's I[ $\left.\omega_{2}\right]$. J. Symbolic Logic, 69(1):94-100, 2004.

[10] W. Mitchell. $I\left[\omega_{2}\right]$ can be the nonstationary ideal on $\operatorname{cof}\left(\omega_{1}\right)$. Trans. Amer. Math. Soc., 361(2):561-601, 2009

[11] E. Schimmerling. Coherent sequences and threads. Adv. Math., 216(1):89-117, 2007. 
[12] S. Shelah. Reflecting stationary sets and successors of singular cardinals. Arch. Math. Logic, 31:25-53, 1991.

[13] S. Shelah. Proper and Improper Forcing. Perspectives in Mathematical Logic. Springer-Verlag, Berlin, second edition, 1998.

[14] R. Solovay. Strongly compact cardinals and the GCH. In Proceedings of the Tarski Symposium (Proc. Sympos. Pure Math., Vol. XXV, Univ. California, Berkeley, Calif., 1971), pages 365372. Amer. Math. Soc., Providence, R.I., 1974.

[15] B. Veličković. Jensen's $\square$ principles and the Novák number of partially ordered sets. J. Symbolic Logic, 51(1):47-58, 1986.

Department of Mathematics, University of North Texas

E-mail address: jkrueger@unt.edu

Department of Mathematical Sciences, Carnegie Mellon University

E-mail address: eschimme@andrew.cmu.edu 